Article

\title{
A Coupled System of Fractional Difference Equations with Nonlocal Fractional Sum Boundary Conditions on the Discrete Half-Line
}

\author{
Jarunee Soontharanon ${ }^{1}$, Saowaluck Chasreechai ${ }^{1, *}$ and Thanin Sitthiwirattham ${ }^{2, *(D)}$ \\ 1 Department of Mathematics, Faculty of Applied Science, King Mongkut's University of Technology \\ North Bangkok, Bangkok 10800, Thailand; jarunee.s@sci.kmutnb.ac.th \\ 2 Mathematics Department, Faculty of Science and Technology, Suan Dusit University, \\ Bangkok 10700, Thailand \\ * Correspondence: saowaluck.c@sci.kmutnb.ac.th (S.C.); thanin_sit@dusit.ac.th (T.S.)
}

Received: 4 February 2019; Accepted: 8 March 2019; Published: 12 March 2019

\begin{abstract}
In this article, we propose a coupled system of fractional difference equations with nonlocal fractional sum boundary conditions on the discrete half-line and study its existence result by using Schauder's fixed point theorem. An example is provided to illustrate the results.
\end{abstract}

Keywords: existence; coupled system of fractional difference equations; fractional sum; discrete half-line

MSC: 39A05; 39A12

\section{Introduction}

Recently, many mathematicians and researchers have extensively studied fractional difference calculus since this subject can be used for describing many problems of real-world phenomena such as mechanical, control systems, flow in porous media, and electrical networks (see [1,2] and the references therein). The basic definitions and properties of fractional difference calculus are given in the book [3]. The applications and developments of the theory can be found in [4-47] and the references cited therein. For example, Ferreira [20] studied the fractional difference equation of order less than one. Goodrich [22] presented the fractional difference equation of order $1<\alpha \leq 2$ with a constant boundary condition. Chen et al. [28] proposed the initial value problem of order less than one. Chen and Zhou [29] studied the antiperiodic boundary value problem of order $1<\alpha \leq 2$. Sitthiwirattham et al. [38] initiated the study of the fractional sum boundary value problem of order $1<\alpha \leq 2$. Sitthiwirattham [40] proposed the sequential fractional difference equation with the fractional sum boundary condition. We observe that these research works are fractional problems containing only one equation.

The study of coupled systems of fractional differential equations is an important topic in this area (see [48-53] and the references cited therein), and a recent example of the application of systems of fractional difference equations is [54].

For the boundary value problems for systems of discrete fractional equations, there are some studies in this area (see [55-60] and the references cited therein).

Pan et al. [55] proposed the system of discrete fractional difference equations as given by:

$$
\begin{aligned}
& -\Delta^{v} y_{1}(t)=f\left(y_{1}(t+v), y_{2}(t+\mu-1)\right), \\
& -\Delta^{\mu} y_{2}(t)=g\left(y_{1}(t+v), y_{2}(t+\mu-1)\right),
\end{aligned}
$$


for $t \in \mathbb{N}_{0, b+1}:=\{0,1,2, \ldots, b+1\}$, with the difference boundary conditions:

$$
\begin{aligned}
& y_{1}(v-2)=\Delta y_{1}(v+b)=0 \\
& y_{2}(\mu-2)=\Delta y_{2}(\mu+b)=0
\end{aligned}
$$

where $b \in \mathbb{N}_{0}:=\mathbb{N} \cup\{0\} ; 1<\mu, v \leq 2 ; 0<\beta \leq 1 ;$ and $f, g: \mathbb{R} \times \mathbb{R} \rightarrow \mathbb{R}$ are continuous functions. $\Delta^{v}$ and $\Delta^{\mu}$ are fractional difference operator of order $v$ and $\mu$, respectively.

In 2015, Goodrich [58] discussed the coupled system of discrete fractional difference equations:

$$
\begin{aligned}
& -\Delta^{-v} x(t)=\lambda_{1} f(t+v-1, y(t+\mu-1)), \quad t \in \mathbb{N}_{0, b+1}, \\
& -\Delta^{-\mu} y(t)=\lambda_{2} g(t+\mu-1, y(t+v-1)),
\end{aligned}
$$

with the nonlinearities satisfying no growth conditions:

$$
\begin{array}{cl}
x(v-2)=H_{1}\left(\sum_{i=1}^{n} a_{i} y\left(\xi_{i}\right)\right), & x(v+b+1)=0, \\
y(\mu-2)=H_{2}\left(\sum_{j=1}^{m} b_{j} x\left(\zeta_{i}\right)\right), & y(\mu+b+1)=0,
\end{array}
$$

where $1<v \leq 2 ; 1<\mu \leq 2 ; \lambda_{1}, \lambda_{2}>0 ;\left\{a_{i}\right\}_{i=1}^{n},\left\{b_{j}\right\}_{j=1}^{m} \subseteq(0, \infty)$; and $H_{1}, H_{2}:[0, \infty) \rightarrow[0, \infty)$ are continuous functions.

In this paper, we considered the coupled system of fractional difference equations:

$$
\left\{\begin{array}{l}
\Delta^{\alpha_{1}} u_{1}(t)=F_{1}\left(t+\alpha_{1}-1, t+\alpha_{2}-1, \Delta^{\beta_{1}} u_{1}\left(t+\alpha_{1}-\beta_{1}\right), u_{2}\left(t+\alpha_{2}-1\right)\right), \\
\Delta^{\alpha_{2}} u_{2}(t)=F_{2}\left(t+\alpha_{1}-1, t+\alpha_{2}-1, \Delta^{\beta_{2}} u_{2}\left(t+\alpha_{2}-\beta_{1}\right), u_{1}\left(t+\alpha_{1}-1\right)\right),
\end{array}\right.
$$

for $t \in \mathbb{N}_{0}$, subject to the nonlocal fractional sum boundary conditions on the discrete half-line $\mathbb{N}_{0}$ :

$$
\left\{\begin{array}{l}
u_{1}\left(\alpha_{1}-2\right)=\phi_{1}\left(u_{1}, u_{2}\right), \\
u_{2}\left(\alpha_{2}-2\right)=\phi_{2}\left(u_{1}, u_{2}\right), \\
\lim _{t \rightarrow \infty} u_{1}\left(t+\alpha_{1}-2\right)=\lambda_{2} \Delta^{-\theta_{2}} g_{2}\left(\eta_{2}+\theta_{2}\right) u_{2}\left(\eta_{2}+\theta_{2}\right), \\
\lim _{t \rightarrow \infty} u_{2}\left(t+\alpha_{2}-2\right)=\lambda_{1} \Delta^{-\theta_{1}} g_{1}\left(\eta_{1}+\theta_{1}\right) u_{1}\left(\eta_{1}+\theta_{1}\right) .
\end{array}\right.
$$

For $i=1,2, \alpha_{i} \in(1,2] ; v_{i}, \gamma_{i}, \theta_{i} \in(0,1] ; \quad \beta_{i} \in\left(\alpha_{i}-1, \alpha_{i}\right) ; \quad \lambda_{1}, \lambda_{2}>0$, and $\eta_{i} \in \mathbb{N}_{\alpha_{i}-1, T+\alpha_{i}-1}$ are given constants; $F_{i} \in C\left(\mathbb{N}_{\alpha_{1}-2} \times \mathbb{N}_{\alpha_{2}-2} \times \mathbb{R}^{2}, \mathbb{R}\right)$ and $g_{i} \in C\left(\mathbb{N}_{\alpha_{i}-2, T+\alpha_{i}}, \mathbb{R}^{+}\right)$are given functions; $\phi_{i}\left(u_{1}, u_{2}\right)$ are given functionals; and $\Delta^{-\theta_{i}}$ are fractional sums of order $\theta_{i}$.

The goal of this study is to show the existence of solutions of the governing problems (5) and (6). The paper is structured as follows. Some definitions and basic lemmas are recalled in Section 2. In Section 3, we prove the existence of solutions of the boundary value problem (5) by employing Schauder's fixed point theorem. Finally, we present an example to illustrate our result in the last section.

\section{Preliminaries}

In what follows, the notation, definitions, and lemmas used in the main results are given.

Definition 1. The generalized falling function is defined by $t^{\underline{\alpha}}:=\frac{\Gamma(t+1)}{\Gamma(t+1-\alpha)}$, for any $t$ and $\alpha$ for which the right-hand side is defined. If $t+1-\alpha$ is a pole of the Gamma function and $t+1$ is not a pole, then $t^{\underline{\alpha}}=0$.

Lemma 1. [4] Assume the falling factorial functions are well defined. If $t \leq r$, then $t^{\underline{\alpha}} \leq r^{\underline{\alpha}}$ for any $\alpha>0$. 
Definition 2. For $\alpha>0$ and $f$ defined on $\mathbb{N}_{a}$, the $\alpha$-order fractional sum of $f$ is defined by:

$$
\Delta^{-\alpha} f(t):=\frac{1}{\Gamma(\alpha)} \sum_{s=a}^{t-\alpha}(t-\sigma(s)) \frac{\alpha-1}{f} f(s)
$$

where $t \in \mathbb{N}_{a+\alpha}$ and $\sigma(s)=s+1$.

Definition 3. For $\alpha>0$ and $f$ defined on $\mathbb{N}_{a}$, the $\alpha$-order Riemann-Liouville fractional difference of $f$ is defined by:

$$
\Delta^{\alpha} f(t):=\Delta^{N} \Delta^{-(N-\alpha)} f(t)=\frac{1}{\Gamma(-\alpha)} \sum_{s=a}^{t+\alpha}(t-\sigma(s)) \frac{-\alpha-1}{-\alpha} f(s),
$$

where $t \in \mathbb{N}_{a+N-\alpha}$ and $N \in \mathbb{N}$ are chosen so that $0 \leq N-1<\alpha \leq N$.

Lemma 2. [4] Let $0 \leq N-1<\alpha \leq N$. Then,

$$
\Delta^{-\alpha} \Delta^{\alpha} y(t)=y(t)+C_{1} t \frac{\alpha-1}{2}+C_{2} t \frac{\alpha-2}{2}+\ldots+C_{N} t \underline{\alpha-N}
$$

for some $C_{i} \in \mathbb{R}$, with $1 \leq i \leq N$.

The following lemma deals with the linear variant of the boundary value problems (5) and (6) and gives a representation of the solution.

Lemma 3. Let $\alpha_{i} \in(1,2], \theta_{i} \in(0,1], \lambda_{1}, \lambda_{2}>0$ and $\eta_{i} \in \mathbb{N}_{\alpha_{i}-1, T+\alpha_{i}-1}$ be given constants, $k_{i} \in C\left(\mathbb{N}_{\alpha_{i}-2}, \mathbb{R}\right)$ and $g_{i} \in C\left(\mathbb{N}_{\alpha_{i}-2, T+\alpha_{i}}, \mathbb{R}^{+}\right)$given functions, and $\phi_{i}\left(u_{1}, u_{2}\right)$ given functionals. For each $i, j \in\{1,2\}$ and $i \neq j$, then the problems:

$$
\begin{aligned}
& \Delta^{\alpha_{i}} u_{i}(t)=k_{i}\left(t+\alpha_{i}-1\right), \quad t \in \mathbb{N}_{0}, \\
& u_{i}\left(\alpha_{i}-2\right)=\phi_{i}\left(u_{1}, u_{2}\right), \\
& \lim _{t \rightarrow \infty} u_{i}\left(t+\alpha_{i}\right)=\lambda_{j} \Delta^{-\theta_{j}} g_{j}\left(\eta_{j}+\theta_{j}\right) u_{j}\left(\eta_{j}+\theta_{j}\right) .
\end{aligned}
$$

have the unique solutions:

$$
\begin{aligned}
& u_{1}\left(t_{1}\right)=t_{1}^{\alpha_{1}-1}\left\{\frac{\lambda_{1}}{\Lambda \Gamma\left(\theta_{1}\right)} \sum_{s=\alpha_{1}-2}^{\eta_{1}}\left(\eta_{1}+\theta_{1}-\sigma(s)\right) \frac{\theta_{1}-1}{g_{1}}(s) s \frac{\alpha_{1}-1}{\mathcal{P}}\left(k_{1}, k_{2}\right)\right. \\
& \left.-\frac{\lambda_{2}}{\Lambda \Gamma\left(\theta_{2}\right)} \sum_{s=\alpha_{2}-2}^{\eta_{2}}\left(\eta_{2}+\theta_{2}-\sigma(s)\right) \frac{\theta_{2}-1}{g_{2}}(s) s \frac{\alpha_{2}-1}{\mathcal{Q}}\left(k_{1}, k_{2}\right)\right\} \\
& +\frac{t \frac{\alpha_{1}-2}{1} \phi_{1}\left(u_{1}, u_{2}\right)}{\Gamma\left(\alpha_{1}\right)}+\frac{1}{\Gamma\left(\alpha_{1}\right)} \sum_{s=0}^{t_{1}-\alpha_{1}}\left(t_{1}-\sigma(s)\right) \frac{\alpha_{1}-1}{2} k_{1}\left(s+\alpha_{1}-1\right), t_{1} \in \mathbb{N}_{\alpha_{1}-2}, \\
& u_{2}\left(t_{2}\right)=t \frac{\alpha_{2}-1}{2}\left\{\frac{\lim _{t_{2} \rightarrow \infty} t_{2}^{\frac{\alpha_{2}-1}{2}}}{\Lambda} \mathcal{P}\left(k_{1}, k_{2}\right)-\frac{\lim _{t_{1} \rightarrow \infty} t_{1}^{\alpha_{1}-1}}{\Lambda} \mathcal{Q}\left(k_{1}, k_{2}\right)\right\} \\
& +\frac{t_{2}^{\alpha_{2}-2} \phi_{2}\left(u_{1}, u_{2}\right)}{\Gamma\left(\alpha_{2}\right)}+\frac{1}{\Gamma\left(\alpha_{2}\right)} \sum_{s=0}^{t_{2}-\alpha_{2}}\left(t_{2}-\sigma(s)\right) \frac{\alpha_{2}-1}{l} k_{2}\left(s+\alpha_{2}-1\right), \quad t_{2} \in \mathbb{N}_{\alpha_{2}-2},
\end{aligned}
$$

provided that both $u_{1}\left(t_{1}\right), u_{2}\left(t_{2}\right)$ are uniformly bounded on $\mathbb{N}_{\alpha_{1}-2}$ and $\mathbb{N}_{\alpha_{2}-2}$, respectively, and:

$$
\Lambda=\frac{\lambda_{2} \lim _{t_{2} \rightarrow \infty} t \frac{\alpha_{2}-1}{2}}{\Gamma\left(\alpha_{2}\right)} \sum_{s=\alpha_{2}-1}^{\eta_{2}}\left(\eta_{2}+\theta_{2}-\sigma(s)\right) \underline{\theta_{2}-1} g_{2}(s) s \underline{\alpha_{2}-1}
$$




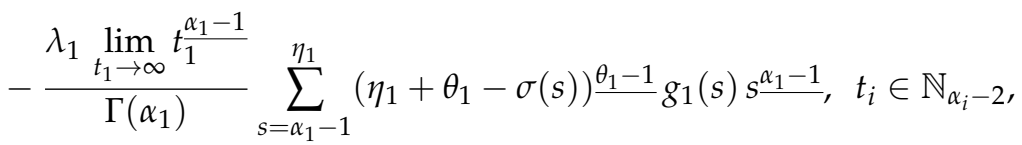

$$
\begin{aligned}
& \mathcal{P}\left(k_{1}, k_{2}\right)=\frac{\lim _{t_{1} \rightarrow \infty} t \frac{\alpha_{1}-2}{1} \phi_{1}\left(u_{1}, u_{2}\right)}{\Gamma\left(\alpha_{1}\right)}-\frac{\lambda_{2} \phi_{2}\left(u_{1}, u_{2}\right)}{\Gamma\left(\alpha_{2}\right) \Gamma\left(\theta_{2}\right)} \sum_{s=\alpha_{2}-2}^{\eta_{2}}\left(\eta_{2}+\theta_{2}-\sigma(s)\right) \frac{\theta_{2}-1}{t_{1}} g_{2}(s) s \frac{\alpha_{2}-2}{2} \\
& +\frac{1}{\Gamma\left(\alpha_{1}\right)} \lim _{t_{1} \rightarrow \infty} \sum_{s=0}^{t_{1}-\alpha_{1}}\left(t_{1}-\sigma(s)\right) \frac{\alpha_{1}-1}{k} k_{1}\left(s+\alpha_{1}-1\right)-\frac{\lambda_{2}}{\Gamma\left(\alpha_{2}\right) \Gamma\left(\theta_{2}\right)} \times
\end{aligned}
$$

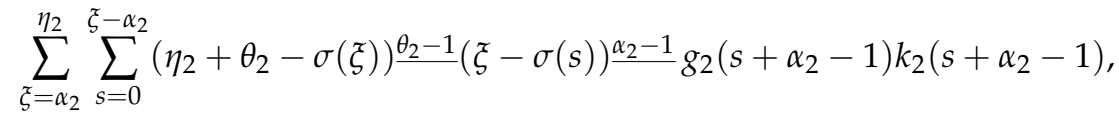

$$
\begin{aligned}
& \mathcal{Q}\left(k_{1}, k_{2}\right)=\frac{\lim _{t_{2} \rightarrow \infty} t \frac{\alpha_{2}-2}{2} \phi_{2}\left(u_{1}, u_{2}\right)}{\Gamma\left(\alpha_{2}\right)}-\frac{\lambda_{1} \phi_{1}\left(u_{1}, u_{2}\right)}{\Gamma\left(\alpha_{1}\right) \Gamma\left(\theta_{1}\right)} \sum_{s=\alpha_{1}-2}^{\eta_{1}}\left(\eta_{1}+\theta_{1}-\sigma(s)\right) \frac{\theta_{1}-1}{g_{1}}(s) s \frac{\alpha_{1}-2}{2} \\
& +\frac{1}{\Gamma\left(\alpha_{2}\right)} \lim _{t_{2} \rightarrow \infty} \sum_{s=0}^{t_{2}-\alpha_{2}}\left(t_{2}-\sigma(s)\right) \frac{\alpha_{2}-1}{k} k_{2}\left(s+\alpha_{2}-1\right)-\frac{\lambda_{1}}{\Gamma\left(\alpha_{1}\right) \Gamma\left(\theta_{1}\right)} \times \\
& \sum_{\xi=\alpha_{1}}^{\eta_{1}} \sum_{s=0}^{\xi-\alpha_{1}}\left(\eta_{1}+\theta_{1}-\sigma(\xi)\right) \frac{\theta_{1}-1}{(\xi-\sigma(s)) \frac{\alpha_{1}-1}{}} g_{1}\left(s+\alpha_{1}-1\right) k_{1}\left(s+\alpha_{1}-1\right) .
\end{aligned}
$$

Proof. For each $i, j \in\{1,2\}$ and $i \neq j$, using Lemma 2 and the fractional sum of order $\alpha \in(1,2]$ for $(7)$, we obtain:

$$
u_{i}\left(t_{i}\right)=C_{1 i} t_{i}^{\alpha_{i}-1}+C_{2 i} t_{i}^{\alpha_{i}-2}+\frac{1}{\Gamma\left(\alpha_{i}\right)} \sum_{s=0}^{t_{i}-\alpha_{i}}\left(t_{i}-\sigma(s)\right) \frac{\alpha_{i}-1}{} k_{i}\left(s+\alpha_{i}-1\right)
$$

for $t_{i} \in \mathbb{N}_{\alpha_{i}-2}$.

By using the boundary condition (8), we find that:

$$
C_{2 i}=\frac{\phi_{i}\left(u_{1}, u_{2}\right)}{\Gamma\left(\alpha_{i}\right)} .
$$

Then, for $t_{i} \in \mathbb{N}_{\alpha_{i}-2}$, we have:

$$
\begin{aligned}
u_{i}\left(t_{i}\right)= & C_{1 i} t_{i}^{\alpha_{i}-1}+\frac{\phi_{i}\left(u_{1}, u_{2}\right)}{\Gamma\left(\alpha_{i}\right)} t_{i}^{\frac{\alpha_{i}-2}{i}} \\
& +\frac{1}{\Gamma\left(\alpha_{i}\right)} \sum_{s=0}^{t_{i}-\alpha_{i}}\left(t_{i}-\sigma(s)\right) \frac{\alpha_{i}-1}{} k_{i}\left(s+\alpha_{i}-1\right) .
\end{aligned}
$$

Taking the fractional sum of order $0<\theta_{i} \leq 1$ for (17), we obtain:

$$
\begin{aligned}
& \Delta^{-\theta_{i}} u_{i}\left(t_{i}\right) \\
= & \frac{C_{1 i}}{\Gamma\left(\theta_{i}\right)} \sum_{s=\alpha_{i}-2}^{t_{i}}\left(t_{i}+\theta-\sigma(s)\right) \frac{\theta_{i}-1}{2} g_{i}(s) s \frac{\alpha_{i}-1}{}+\frac{\phi_{i}\left(u_{1}, u_{2}\right)}{\Gamma\left(\alpha_{i}\right)} \times \\
& \sum_{s=\alpha_{i}-2}^{t_{i}}\left(t_{i}+\theta_{i}-\sigma(s)\right) \frac{\theta_{i}-1}{g_{i}} g_{i}(s) s \frac{\alpha_{i}-2}{}+\frac{1}{\Gamma\left(\theta_{i}\right) \Gamma\left(\alpha_{i}\right)} \sum_{\xi=\alpha_{i}}^{t_{i}} \sum_{s=0}^{\xi-\alpha_{i}}\left(t_{i}+\theta_{i}-\sigma(\xi)\right) \frac{\theta_{i}-1}{} \times \\
& (\xi-\sigma(s)) \frac{\alpha_{i}-1}{2} g_{i}\left(s+\alpha_{i}-1\right) k_{i}\left(s+\alpha_{i}-1\right),
\end{aligned}
$$

for $t_{i} \in \mathbb{N}_{\alpha_{i}-2}$. 
Employing the boundary condition (9), this implies that:

$$
\begin{aligned}
& C_{11} \lim _{t_{1} \rightarrow \infty} t \frac{\alpha_{1}-1}{1}+\frac{\phi_{1}\left(u_{1}, u_{2}\right)}{\Gamma\left(\alpha_{1}\right)} \lim _{t_{1} \rightarrow \infty} t \frac{\alpha_{1}-2}{1}+\frac{\lim _{t_{1} \rightarrow \infty}}{\Gamma\left(\alpha_{1}\right)} \sum_{s=0}^{t_{1}-\alpha_{1}}\left(t_{i}-\sigma(s)\right) \frac{\alpha_{1}-1}{2} k_{1}\left(s+\alpha_{1}-1\right) \\
= & \frac{\lambda_{2} C_{12}}{\Gamma\left(\theta_{2}\right)} \sum_{s=\alpha_{2}-1}^{\eta_{2}}\left(\eta_{2}+\theta_{2}-\sigma(s)\right) \frac{\theta_{2}-1}{g_{2}} g_{2}(s) s \frac{\alpha_{2}-1}{\underline{\alpha_{2}}} \\
& +\frac{\lambda_{2} \phi_{2}\left(u_{1}, u_{2}\right)}{\Gamma\left(\alpha_{2}\right) \Gamma\left(\theta_{2}\right)} \sum_{s=\alpha_{2}-2}^{\eta_{2}}\left(\eta_{2}+\theta_{2}-\sigma(s)\right) \frac{\theta_{2}-1}{g} g_{2}(s) s \frac{\alpha_{2}-2}{\eta_{2}} \\
& +\frac{\lambda_{2}}{\Gamma\left(\alpha_{2}\right) \Gamma\left(\theta_{2}\right)} \sum_{\xi=\alpha_{2}}^{\eta_{2}} \sum_{s=0}^{\xi-\alpha_{2}}\left(\eta_{2}+\theta_{2}-\sigma(\xi)\right) \frac{\theta_{2}-1}{(\xi-\sigma(s)) \frac{\alpha_{2}-1}{2}} g_{2}\left(s+\alpha_{2}-1\right) k_{2}\left(s+\alpha_{2}-1\right),
\end{aligned}
$$

and:

$$
\begin{aligned}
& C_{12} \lim _{t_{2} \rightarrow \infty} t \frac{\alpha_{2}-1}{2}+\frac{\phi_{2}\left(u_{1}, u_{2}\right)}{\Gamma\left(\alpha_{2}\right)} \lim _{t_{2} \rightarrow \infty} t \frac{\alpha_{2}-2}{2}+\frac{1}{\Gamma\left(\alpha_{2}\right)} \lim _{t_{2} \rightarrow \infty} \sum_{s=0}^{t_{2}-\alpha_{2}}\left(t_{2}-\sigma(s)\right) \underline{\alpha_{2}-1} k_{2}\left(s+\alpha_{2}-1\right) \\
& =\frac{\lambda_{1} C_{11}}{\Gamma\left(\theta_{1}\right)} \sum_{s=\alpha_{1}-1}^{\eta_{1}}\left(\eta_{1}+\theta_{1}-\sigma(s)\right) \frac{\theta_{1}-1}{2} g_{1}(s) s \underline{\alpha_{1}-1} \\
& +\frac{\lambda_{1} \phi_{1}\left(u_{1}, u_{2}\right)}{\Gamma\left(\alpha_{1}\right) \Gamma\left(\theta_{1}\right)} \sum_{s=\alpha_{1}-2}^{\eta_{1}}\left(\eta_{1}+\theta_{1}-\sigma(s)\right) \frac{\theta_{1}-1}{} g_{1}(s) s \underline{\alpha_{1}-2}
\end{aligned}
$$

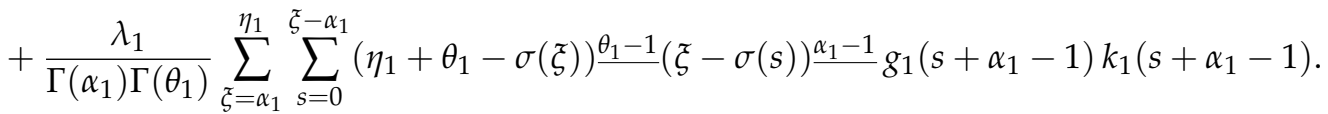

After solving the system of Equations (19) and (20), we obtain:

$$
\begin{aligned}
C_{11}= & \frac{\lambda_{1}}{\Lambda \Gamma\left(\theta_{1}\right)} \sum_{s=\alpha_{1}-2}^{\eta_{1}}\left(\eta_{1}+\theta_{1}-\sigma(s)\right) \frac{\theta_{1}-1}{2} g_{1}(s) s \frac{\alpha_{1}-1}{\mathcal{P}}\left(k_{1}, k_{2}\right) \\
& -\frac{\lambda_{2}}{\Lambda \Gamma\left(\theta_{2}\right)} \sum_{s=\alpha_{2}-2}^{\eta_{2}}\left(\eta_{2}+\theta_{2}-\sigma(s)\right) \frac{\theta_{2}-1}{g} g_{2}(s) s \frac{\alpha_{2}-1}{\underline{Q}} \mathcal{Q}\left(k_{1}, k_{2}\right),
\end{aligned}
$$

and:

$$
C_{12}=\frac{\lim _{t_{2} \rightarrow \infty} t \frac{\alpha_{2}-1}{2}}{\Lambda} \mathcal{P}\left(k_{1}, k_{2}\right)-\frac{\lim _{t_{1} \rightarrow \infty} t \frac{\alpha_{1}-1}{1}}{\Lambda} \mathcal{Q}\left(k_{1}, k_{2}\right),
$$

where $\Lambda, \mathcal{P}\left(k_{1}, k_{2}\right)$ and $\mathcal{Q}\left(k_{1}, k_{2}\right)$ are defined as (12)-(14), respectively.

The following lemma deals with the solutions $u_{i}\left(t_{i}\right), i=1,2$ of the problems (7)-(9), and $\Delta^{\beta_{i}} u_{i}\left(t_{i}-\beta_{i}+1\right)$ are uniformly bounded on $\mathbb{N}_{\alpha_{i}-2}, \beta_{i} \in\left(\alpha_{i}-1, \alpha_{i}\right)$.

Lemma 4. For each $i, j \in\{1,2\}$ and $i \neq j$, let $k_{i} \in C\left(\mathbb{N}_{\alpha_{i}-2}, \mathbb{R}\right)$ and $g_{i} \in C\left(\mathbb{N}_{\alpha_{i}-2}, \mathbb{R}^{+}\right)$be given functions, $\phi_{i}\left(u_{1}, u_{2}\right)$ be given functionals, $\rho_{i}>\max \left\{\beta_{i}-\alpha_{i}\right\}, \beta_{i} \in\left(\alpha_{i}-1, \alpha_{i}\right)$, and $0<g_{i} \leq g_{i}\left(s_{i}\right) \leq G_{i}$, for each $s_{i} \in \mathbb{N}_{\alpha_{i}-2, T+\alpha_{i}}$.

The solution $u_{i}\left(t_{i}\right)$ of the problems (7)-(9) and $\Delta^{\beta_{i}} u_{i}\left(t_{i}-\beta_{i}+1\right)$ are uniformly bounded on $\mathbb{N}_{\alpha_{i}-2}$, if and only if $u_{i}\left(t_{i}\right)$ and $\Delta^{\beta_{i}} u_{i}\left(t_{i}-\beta_{i}+1\right)$ satisfy the following properties:

$\left(A_{1}\right)$ There exist constants $M_{1}, N_{1}, m_{1}, n_{1}>0$ such that, for $u_{1}$ and $\Delta^{\beta_{1}} u_{1}$,

$$
\left|k_{i}\left(t_{i}\right)\right| \leq M_{1} e^{-m_{1}\left(2 t_{1}+t_{2}\right)},
$$




$$
\left|\phi_{i}\left(u_{1}, u_{2}\right)\right| \leq N_{1}\left(t_{1}+\rho_{1}\right) \underline{\rho_{1}} e^{-n_{1}\left(t_{1}+1\right)} .
$$

$\left(A_{2}\right)$ There exist constants $M_{2}, N_{2}, m_{2}, n_{2}>0$ such that, for $u_{2}$ and $\Delta^{\beta_{2}} u_{2}$,

$$
\begin{aligned}
\left|k_{i}\left(t_{i}\right)\right| & \leq M_{2}\left(t_{2}+\rho_{2}\right) \underline{\rho_{2}} e^{-m_{2}\left(t_{1}+2 t_{2}\right)}, \\
\left|\phi_{i}\left(u_{1}, u_{2}\right)\right| & \leq N_{2}\left(t_{1}+\rho_{1}\right) \underline{\rho_{1}}\left[\left(t_{2}+\rho_{2}\right)^{\frac{\rho_{2}}{2}}\right]^{2} e^{-n_{2}\left(t_{2}+1\right)} .
\end{aligned}
$$

$\left(A_{3}\right)$ There exist constants $\Omega_{i}>0, i=1,2$ such that,

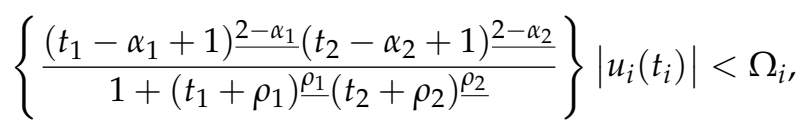

$$
\begin{aligned}
& \left\{\frac{\left(t_{1}-\alpha_{1}+1\right)^{2-\alpha_{1}}\left(t_{2}-\alpha_{2}+1\right)^{\frac{2-\alpha_{2}}{2}}}{1+\left(t_{1}+\rho_{1}\right)^{\underline{\rho_{1}}}\left(t_{2}+\rho_{2}\right)^{\underline{\rho_{2}}}}\right\}\left|\Delta^{\beta_{i}} u_{i}\left(t_{i}\right)\right|<\Omega_{i} \text {. }
\end{aligned}
$$

Proof. Firstly, taking the fractional difference of order $\alpha_{i}-1<\beta_{i}<\alpha_{i}, i=1,2$ for (10) and (11), we obtain:

$$
\begin{aligned}
& \Delta^{\beta_{1}} u_{1}\left(t_{1}\right) \\
& =\frac{1}{\Gamma\left(-\beta_{1}\right)} \sum_{s=\alpha_{1}-1}^{t_{1}+1}\left(t_{1}-\beta_{1}+1-\sigma(s)\right) \frac{-\beta_{1}-1}{\underline{\alpha_{1}}} \frac{\alpha_{1}-1}{} \times\left\{\frac{\lambda_{1}}{\Lambda \Gamma\left(\theta_{1}\right)} \times\right. \\
& \sum_{s=\alpha_{1}-2}^{\eta_{1}}\left(\eta_{1}+\theta_{1}-\sigma(s)\right) \frac{\theta_{1}-1}{g} g_{1}(s) s \frac{\alpha_{1}-1}{} \mathcal{P}\left(k_{1}, k_{2}\right)-\frac{\lambda_{2}}{\Lambda \Gamma\left(\theta_{2}\right)} \times \\
& \left.\sum_{s=\alpha_{2}-2}^{\eta_{2}}\left(\eta_{2}+\theta_{2}-\sigma(s)\right) \frac{\theta_{2}-1}{g_{2}}(s) s \frac{\alpha_{2}-1}{Q} \mathcal{Q}\left(k_{1}, k_{2}\right)\right\} \\
& +\frac{\phi_{1}\left(u_{1}, u_{2}\right)}{\Gamma\left(-\beta_{1}\right) \Gamma\left(\alpha_{1}\right)} \sum_{s=\alpha_{1}-2}^{t_{1}+1}\left(t_{1}-\beta_{1}+1-\sigma(s)\right) \underline{-\beta_{1}-1} s \underline{\alpha_{1}-2} \\
& +\frac{1}{\Gamma\left(-\beta_{1}\right) \Gamma\left(\alpha_{1}\right)} \sum_{\xi=\alpha_{1}}^{t_{1}+1} \sum_{s=0}^{\xi-\alpha_{1}}\left(t_{1}-\beta_{1}+1-\sigma(s)\right) \frac{-\beta_{1}-1}{}(\xi-\sigma(s)) \frac{\alpha_{1}-1}{k} k_{1}\left(s+\alpha_{1}-1\right),
\end{aligned}
$$

and:

$$
\begin{aligned}
& \Delta^{\beta_{2}} u_{2}\left(t_{2}\right) \\
& =\frac{1}{\Gamma\left(-\beta_{2}\right)} \sum_{s=\alpha_{2}-1}^{t_{2}+1}\left(t_{2}-\beta_{2}+1-\sigma(s)\right) \frac{-\beta_{2}-1}{S} s \frac{\alpha_{2}-1}{\alpha_{2}} \times\left\{\frac{\lim _{2} \rightarrow \infty}{\Lambda} \frac{\alpha_{2}-1}{2} \mathcal{P}\left(k_{1}, k_{2}\right)\right. \\
& \left.-\frac{\lim _{t_{1} \rightarrow \infty} t \frac{\alpha_{1}-1}{1}}{\Lambda} \mathcal{Q}\left(k_{1}, k_{2}\right)\right\}+\frac{\phi_{2}\left(u_{1}, u_{2}\right)}{\Gamma\left(-\beta_{2}\right) \Gamma\left(\alpha_{2}\right)} \sum_{s=\alpha_{2}-2}^{t_{2}+1}\left(t_{2}-\beta_{1}+1-\sigma(s)\right) \frac{-\beta_{2}-1}{s} s \frac{\alpha_{2}-2}{2} \\
& +\frac{1}{\Gamma\left(-\beta_{2}\right) \Gamma\left(\alpha_{2}\right)} \sum_{\xi=\alpha_{2}}^{t_{2}+1} \sum_{s=0}^{\xi-\alpha_{2}}\left(t_{2}-\beta_{2}+1-\sigma(s)\right) \frac{-\beta_{2}-1}{}(\xi-\sigma(s)) \frac{\alpha_{2}-1}{k} k_{2}\left(s+\alpha_{2}-1\right) .
\end{aligned}
$$

If $u_{i}\left(t_{i}\right)$ and $\Delta^{\beta_{i}} u_{i}\left(t_{i}-\beta_{i}+1\right)$ are uniformly bounded on $\mathbb{N}_{\alpha_{i}-2}$, we have:

$$
|\Lambda| \leq \mid \frac{\lambda_{2} \lim _{t_{2} \rightarrow \infty} t \frac{\alpha_{2}-1}{2}}{\Gamma\left(\alpha_{2}\right)} \sum_{s=\alpha_{2}-1}^{\eta_{2}}\left(\eta_{2}+\theta_{2}-\sigma(s)\right) \frac{\theta_{2}-1}{2} g_{2}(s) s \underline{\alpha_{2}-1}
$$




$$
\begin{gathered}
-\frac{\lambda_{1} \lim _{t_{1} \rightarrow \infty} t^{\frac{\alpha_{1}-1}{1}}}{\Gamma\left(\alpha_{1}\right)} \sum_{s=\alpha_{1}-1}^{\eta_{1}}\left(\eta_{1}+\theta_{1}-\sigma(s)\right) \frac{\theta_{1}-1}{g_{1}} g_{1}(s) s \frac{\alpha_{1}-1}{} \mid \\
\leq \max \left\{\left|\lim _{t_{2} \rightarrow \infty} t \frac{\alpha_{2}-1}{2}\left(\eta_{2}+\theta_{2}-\alpha_{2}\right) \frac{\theta_{2}-1}{G_{2}} \lambda_{2} \mathcal{A}_{2}\right|,\right. \\
\left.\left|\lim _{t_{1} \rightarrow \infty} t \frac{\alpha_{1}-1}{1}\left(\eta_{1}+\theta_{1}-\alpha_{1}\right) \frac{\theta_{1}-1}{2} G_{1} \lambda_{1} \mathcal{A}_{1}\right|\right\} .
\end{gathered}
$$

Furthermore, considering $u_{1}\left(t_{i}\right)$ and $\Delta^{\beta_{1}} u_{i}\left(t_{i}\right)$, we obtain:

$$
\left|k_{i}\left(t_{i}\right)\right|< \begin{cases}M_{1} e^{1-\left(t_{1}+t_{2}\right)} & , \text { for } u_{1}\left(t_{1}\right) \\ M_{1} e^{-\left(2 t_{1}+t_{2}\right)} & , \text { for } \Delta^{\beta_{1}} u_{1}\left(t_{1}-\beta_{1}+1\right) \\ M_{2}\left(t_{2}+\rho_{2}\right) \frac{\rho_{2}}{2} e^{-\left(t_{1}+t_{2}\right)} & , \text { for } u_{2}\left(t_{2}\right) \\ M_{2}\left(t_{2}+\rho_{2}\right) \frac{\rho_{2}}{2} e^{-\left(t_{1}+2 t_{2}\right)} & , \text { for } \Delta^{\beta_{2}} u_{2}\left(t_{2}-\beta_{2}+1\right)\end{cases}
$$

and:

$$
\left|\phi_{i}\left(t_{1}, t_{2}\right)\right|< \begin{cases}N_{1}\left(t_{1}+\rho_{1}+1\right) \underline{\rho_{1}} & , \text { for } u_{1}\left(t_{1}\right) \\ N_{1}\left(t_{1}+\rho_{1}\right) \frac{\rho_{1}}{e^{-\left(t_{1}+1\right)}} & , \text { for } \Delta^{\beta_{1}} u_{1}\left(t_{1}-\beta_{1}+1\right) \\ N_{2}\left(t_{1}+\rho_{1}\right) \underline{\rho_{1}}\left[\left(t_{2}+\rho_{2}+1\right) \underline{\rho_{2}}\right]^{2} & , \text { for } u_{2}\left(t_{2}\right) \\ N_{2}\left(t_{1}+\rho_{1}\right) \underline{\rho_{1}}\left[\left(t_{2}+\rho_{2}\right) \underline{\rho_{2}}\right]^{2} e^{-\left(t_{2}+1\right)} & , \text { for } \Delta^{\beta_{2}} u_{2}\left(t_{2}-\beta_{2}+1\right)\end{cases}
$$

where:

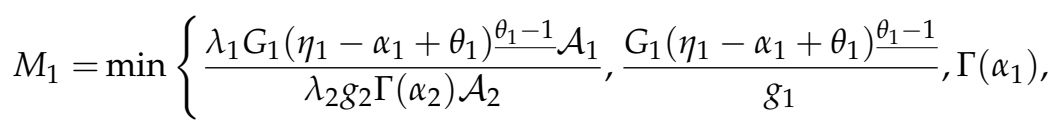

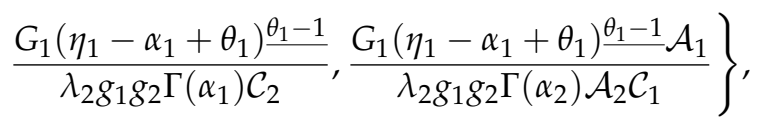

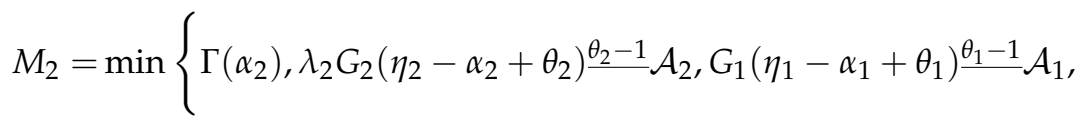

$$
\begin{aligned}
& \left.\frac{G_{1}\left(\eta_{1}-\alpha_{1}+\theta_{1}\right) \frac{\theta_{1}-1}{\mathcal{A}_{1}}}{g_{1} \mathcal{C}_{1}}, \frac{G_{2}\left(\eta_{2}-\alpha_{2}+\theta_{2}\right) \stackrel{\theta_{2}-1}{\mathcal{A}_{2}}}{g_{2} \mathcal{C}_{2}}\right\},
\end{aligned}
$$

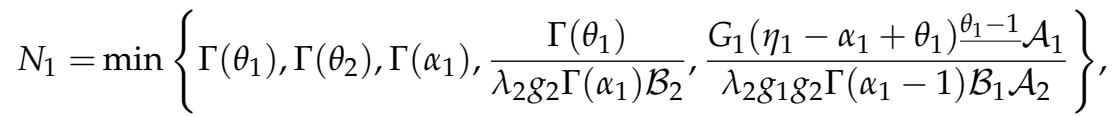

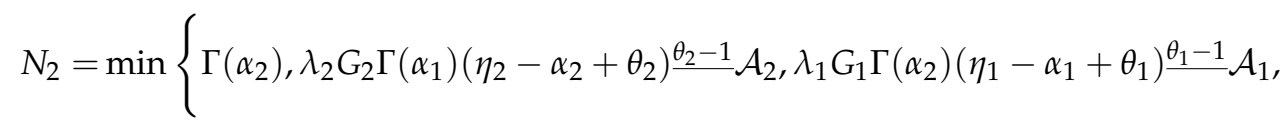

$$
\begin{aligned}
& \left.\frac{G_{2}\left(\eta_{2}-\alpha_{2}+\theta_{2}\right) \frac{\theta_{2}-1}{\mathcal{A}_{2}}}{g_{2} \mathcal{B}_{2}}, \frac{G_{1}\left(\eta_{1}-\alpha_{1}+\theta_{1}\right) \frac{\theta_{1}-1}{\mathcal{A}_{1}}}{g_{1} \mathcal{B}_{1}}\right\} \text {. }
\end{aligned}
$$

Consequently, the conditions $(A 1)$ and $(A 2)$ hold.

We next show that the condition $(A 3)$ holds. By using the conditions $(A 1)$ and $(A 2)$, we obtain:

$$
\begin{aligned}
\left|u_{i}\left(t_{i}\right)\right| \leq t \frac{\alpha_{1}}{1} t \frac{\alpha_{2}}{2} \Omega_{i} & <t \frac{\alpha_{1}+\rho_{1}-1}{1} t \frac{\alpha_{2}+\rho_{2}-1}{2} \Omega_{i} \\
& <\left[\frac{1+t \frac{\rho_{1}+2}{1} t \frac{\rho_{2}+2}{2}}{\left(t_{1}-\alpha_{1}+1\right) \frac{2-\alpha_{1}}{2}\left(t_{2}-\alpha_{2}+1\right) \frac{2-\alpha_{2}}{2}}\right] \Omega_{i}
\end{aligned}
$$

and: 


$$
\begin{aligned}
\left|\Delta^{\beta_{i}} u_{i}\left(t_{i}-\beta_{i}+1\right)\right| & \leq\left(t_{i}-\alpha_{i}-\beta_{i}+1\right) \frac{-\beta_{i}}{t} t_{j}^{\frac{\alpha_{j}}{j}} \Omega_{i}<t_{i}^{\frac{\alpha_{i}}{\alpha_{j}}} t_{j}^{\frac{\alpha_{j}}{j}} \Omega_{i} \\
& <\left[\frac{1+t_{1}^{\rho_{1}+2} t_{2}}{\left(t_{1}-\alpha_{1}+1\right) \frac{2-\alpha_{1}}{2}\left(t_{2}-\alpha_{2}+1\right) \frac{2-\alpha_{2}}{2}}\right] \Omega_{i}, \quad i \neq j=1,2
\end{aligned}
$$

where:

$$
\begin{aligned}
& \Omega_{1}=\max \left\{\frac{N_{1}}{\Gamma\left(\theta_{1}\right)}+M_{1}+\frac{\lambda_{2} G_{2} \Gamma\left(\alpha_{1}\right)\left(\eta_{2}-\alpha_{2}+\theta_{2}\right) \frac{\theta_{2}-1}{\Gamma\left(\theta_{2}\right)} \times}{N_{2}}\right), \\
&\left(N_{2} \mathcal{B}_{2}+\frac{M_{2} \mathcal{C}_{2}}{\lambda_{1} G_{1}\left(\eta_{1}-\alpha_{1}+\theta_{1}\right) \frac{\theta_{1}-1}{\mathcal{A}_{1}}}\right) \\
&\left.\left.\frac{N_{2}}{\Gamma\left(\theta_{2}\right)}+M_{2}+\frac{\lambda_{1} G_{1} \Gamma\left(\alpha_{2}\right)\left(\eta_{1}-\alpha_{1}+\theta_{1}\right) \frac{\theta_{1}-1}{\Gamma\left(\theta_{1}\right)} \times}{M_{1} \mathcal{C}_{1}}\right)\right\} \\
&+ \frac{1}{\Gamma\left(\alpha_{1}\right)}\left(N_{1} \mathcal{B}_{1}+\frac{1}{\left.\lambda_{2} G_{2}\left(\eta_{2}-\alpha_{2}+\theta_{2}\right) \frac{\theta_{2}-1}{\mathcal{A}_{2}}\right),}\right. \\
& \Omega_{2}=\max \left\{\frac{1}{G_{2}\left(\eta_{2}-\alpha_{2}+\theta_{2}\right) \frac{\theta_{2}-1}{\mathcal{A}_{2}}}\left[\frac{M_{1}+N_{1}}{\Gamma\left(\alpha_{1}\right) \lambda_{2}}+\frac{1}{\Gamma\left(\theta_{2}\right) \mathcal{A}_{2}}\left(N_{2} \mathcal{B}_{2}+M_{2} \mathcal{C}_{2}\right)\right],\right. \\
&\left.\quad \frac{1}{G_{1}\left(\eta_{1}-\alpha_{1}+\theta_{1}\right) \frac{\theta_{1}-1}{\mathcal{A}_{1}}}\left[\frac{M_{2}+N_{2}}{\Gamma\left(\alpha_{2}\right) \lambda_{1}}+\frac{1}{\Gamma\left(\theta_{1}\right) \mathcal{A}_{1}}\left(N_{1} \mathcal{B}_{1}+M_{1} \mathcal{C}_{1}\right)\right]\right\} \\
&+\frac{1}{\Gamma\left(\alpha_{2}\right)}\left(M_{2}+N_{2}\right),
\end{aligned}
$$

with

$$
\begin{aligned}
& \mathcal{A}_{i}={ }_{2} F_{1}\left(\alpha_{i}, \alpha_{i}-\eta_{i}-1 ; \alpha_{i}-\eta_{i}-\theta_{i} ; 1\right) \\
& \mathcal{B}_{i}={ }_{2} F_{1}\left(\alpha_{i}-1, \alpha_{i}-\eta_{i}-1 ; \alpha_{i}-\eta_{i}-\theta_{i}-1 ; 1\right) \\
& \mathcal{C}_{i}={ }_{2} F_{1}\left(\alpha_{i}+1, \alpha_{i}-\eta_{i} ; \alpha_{i}-\eta_{i}-\theta_{i}+1 ; 1\right) .
\end{aligned}
$$

Therefore, the condition $(A 3)$ holds.

Finally, if the conditions $(A 1)-(A 3)$ hold, it is clear that $u_{i}\left(t_{i}\right)$ and $\Delta^{\beta_{i}} u_{i}\left(t_{i}-\beta_{i}+1\right)$ are uniformly bounded on $\mathbb{N}_{\alpha_{i}-2}$. Our proof is complete. and (6).

We next provide the following theorems used for proving the existence result for the problems (5)

Theorem 1. (Arzelá-Ascoli theorem [61])

A set of functions in $C[a, b]$ with the sup norm is relatively compact if and only if it is uniformly bounded and equicontinuous on $[a, b]$.

Theorem 2. [61] If a set is closed and relatively compact, then it is compact.

Theorem 3. (Schauder's fixed point theorem [61])

If $S$ is a convex compact subset of a normed space, every continuous mapping of $S$ into itself has a fixed point. 


\section{Main Result}

In this section, we aim to establish the existence result for the problems (5) and (6). To accomplish this, we let $\mathcal{C}_{i}=C\left(\mathbb{N}_{\alpha_{i}-2}, \mathbb{R}\right)$ be a Banach space of all functions on $\mathbb{N}_{\alpha_{i}-2}$, for each $i, j \in\{1,2\}$ and $i \neq j$. Obviously, the product spaces:

$$
\begin{aligned}
\mathcal{U}_{i}=\{ & \left(u_{1}, u_{2}\right) \in \mathcal{C}_{1} \times \mathcal{C}_{2}: \Delta^{\beta_{i}} u_{i}\left(t_{i}-\beta_{i}+1\right) \in \mathcal{C}_{i} \text { and } \chi\left|u_{j}\left(t_{j}\right)\right|, \\
& \left.\chi\left|\Delta^{\beta_{i}} u_{i}\left(t_{i}-\beta_{i}+1\right)\right| \text { are bounded on } \mathbb{N}_{\alpha_{j}-2}, \mathbb{N}_{\alpha_{i}-2}, \text { respectively, }\right\}
\end{aligned}
$$

is also the Banach space endowed with the norm defined by:

$$
\left\|\left(u_{1}, u_{2}\right)\right\|_{\mathcal{U}_{i}}=\left\|\Delta^{\beta_{i}} u_{i}\right\|_{\mathcal{C}_{i}}+\left\|u_{j}\right\|_{\mathcal{C}_{j}}
$$

where:

$$
\left\|\Delta^{\beta_{i}} u_{i}\right\|_{\mathcal{C}_{i}}=\max _{t_{i} \in \mathbb{N}_{\alpha_{i}-2}} \chi\left|\Delta^{\beta_{i}} u_{i}\left(t_{i}-\beta_{i}+1, t_{j}\right)\right| \text { and }\left\|u_{j}\right\|_{\mathcal{C}_{j}}=\max _{t_{j} \in \mathbb{N}_{\alpha_{j}-2}} \chi\left|u_{j}\left(t_{i}, t_{j}\right)\right|,
$$

with for $\rho_{i}>\max \left\{\beta_{i}-\alpha_{i}\right\}$ and $\beta_{i} \in\left(\alpha_{i}-1, \alpha_{i}\right)$,

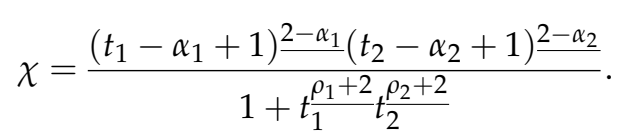

Let $\mathcal{U}=\mathcal{U}_{1} \cap \mathcal{U}_{2} ;$ clearly, the space $\left(\mathcal{U},\left\|\left(u_{1}, u_{2}\right)\right\|_{\mathcal{U}}\right)$ is the Banach space with the norm:

$$
\left\|\left(u_{1}, u_{2}\right)\right\|_{\mathcal{U}}=\max \left\{\left\|\left(u_{1}, u_{2}\right)\right\|_{\mathcal{U}_{1}},\left\|\left(u_{1}, u_{2}\right)\right\|_{\mathcal{U}_{2}}\right\} .
$$

Next, we define the operator $\mathcal{F}: \mathcal{U} \rightarrow \mathcal{U}$ by:

$$
\left(\mathcal{F}\left(u_{1}, u_{2}\right)\right)\left(t_{1}, t_{2}\right)=\left(\left(\mathcal{F}_{1}\left(u_{1}, u_{2}\right)\right)\left(t_{1}, t_{2}\right),\left(\mathcal{F}_{2}\left(u_{1}, u_{2}\right)\right)\left(t_{1}, t_{2}\right)\right),
$$

and:

$$
\begin{aligned}
& \left(\mathcal{F}_{1}\left(u_{1}, u_{2}\right)\right)\left(t_{1}, t_{2}\right)=\frac{t_{1}^{\alpha_{1}-1}}{\Lambda}\left\{\frac{\lambda_{1}}{\Gamma\left(\theta_{1}\right)} \sum_{s=\alpha_{1}-2}^{\eta_{1}}\left(\eta_{1}+\theta_{1}-\sigma(s)\right) \frac{\theta_{1}-1}{g_{1}}(s) s \frac{\alpha_{1}-1}{\mathcal{P}}\left(F_{1}, F_{2}\right)\right. \\
& \left.-\frac{\lambda_{2}}{\Gamma\left(\theta_{2}\right)} \sum_{s=\alpha_{2}-2}^{\eta_{2}}\left(\eta_{2}+\theta_{2}-\sigma(s)\right) \underline{\theta_{2}-1} g_{2}(s) s \underline{\alpha_{2}-1} \mathcal{Q}\left(F_{1}, F_{2}\right)\right\} \\
& +\frac{t^{\frac{\alpha_{1}-2}{1}} \phi_{1}\left(u_{1}, u_{2}\right)}{\Gamma\left(\alpha_{1}\right)}+\frac{1}{\Gamma\left(\alpha_{1}\right)} \sum_{s=\alpha_{1}-1}^{t_{1}-1}\left(t_{1}+\alpha_{1}-1-\sigma(s)\right) \frac{\alpha_{1}-1}{} \times \\
& F_{1}\left(s, t_{2}, \Delta^{\beta_{1}} u_{1}\left(s-\beta_{1}+1\right), u_{2}\left(t_{2}\right)\right), t_{i} \in \mathbb{N}_{\alpha_{i}-2} \text {, } \\
& \left(\mathcal{F}_{2}\left(u_{1}, u_{2}\right)\right)\left(t_{1}, t_{2}\right)=\frac{t \frac{\alpha_{2}-1}{2}}{\Lambda}\left\{\lim _{t_{2} \rightarrow \infty} t \frac{\alpha_{2}-1}{2} \mathcal{P}\left(F_{1}, F_{2}\right)-\lim _{t_{1} \rightarrow \infty} t \frac{\alpha_{1}-1}{1} \mathcal{Q}\left(F_{1}, F_{2}\right)\right\} \\
& +\frac{t_{2}^{\alpha_{2}-2} \phi_{2}\left(u_{1}, u_{2}\right)}{\Gamma\left(\alpha_{2}\right)}+\frac{1}{\Gamma\left(\alpha_{2}\right)} \sum_{s=\alpha_{2}-1}^{t_{2}-1}\left(t_{2}+\alpha_{2}-1-\sigma(s)\right) \frac{\alpha_{2}-1}{} \times \\
& F_{2}\left(t_{1}, s, u_{1}\left(t_{1}\right), \Delta^{\beta_{2}} u_{2}\left(s-\beta_{2}+1\right)\right), t_{i} \in \mathbb{N}_{\alpha_{i}-2} \text {, }
\end{aligned}
$$


where $\Lambda$ is defined as (12), and:

$$
\begin{aligned}
& \mathcal{P}\left(F_{1}, F_{2}\right)=\frac{\lim _{t_{1} \rightarrow \infty} t \frac{\alpha_{1}-2}{1} \phi_{1}\left(u_{1}, u_{2}\right)}{\Gamma\left(\alpha_{1}\right)}-\frac{\lambda_{2} \phi_{2}\left(u_{1}, u_{2}\right)}{\Gamma\left(\alpha_{2}\right) \Gamma\left(\theta_{2}\right)} \sum_{s=\alpha_{2}-2}^{\eta_{2}}\left(\eta_{2}+\theta_{2}-\sigma(s)\right) \underline{\theta_{2}-1} g_{2}(s) s \frac{\alpha_{2}-2}{t_{1}-1} \\
& +\frac{1}{\Gamma\left(\alpha_{1}\right)} \lim _{t_{1} \rightarrow \infty} \sum_{s=\alpha_{1}-1}^{t_{1}-1}\left(t_{1}+\alpha_{1}-1-\sigma(s)\right) \frac{\alpha_{1}-1}{\frac{1}{2}} F_{1}\left(s, t_{2}, \Delta^{\beta_{1}} u_{1}\left(s-\beta_{1}+1\right), u_{2}\left(t_{2}\right)\right) \\
& -\frac{\lambda_{2}}{\Gamma\left(\alpha_{2}\right) \Gamma\left(\theta_{2}\right)} \sum_{\xi=\alpha_{2}}^{\eta_{2}} \sum_{s=\alpha_{2}-1}^{\xi-1}\left(\eta_{2}+\theta_{2}-\sigma(\xi)\right) \frac{\theta_{2}-1}{\xi}\left(\xi+\alpha_{2}-1-\sigma(s)\right) \frac{\alpha_{2}-1}{} \times \\
& g_{2}(s) F_{2}\left(t_{1}, s, u_{1}\left(t_{1}\right), \Delta^{\beta_{2}} u_{2}\left(s-\beta_{2}+1\right)\right) \text {, } \\
& \mathcal{Q}\left(F_{1}, F_{2}\right)=\frac{\lim _{t_{2} \rightarrow \infty} t \frac{\alpha_{2}-2}{2} \phi_{2}\left(u_{1}, u_{2}\right)}{\Gamma\left(\alpha_{2}\right)}-\frac{\lambda_{1} \phi_{1}\left(u_{1}, u_{2}\right)}{\Gamma\left(\alpha_{1}\right) \Gamma\left(\theta_{1}\right)} \sum_{s=\alpha_{1}-2}^{\eta_{1}}\left(\eta_{1}+\theta_{1}-\sigma(s)\right) \frac{\theta_{1}-1}{g} g_{1}(s) s \frac{\alpha_{1}-2}{2} \\
& +\frac{1}{\Gamma\left(\alpha_{2}\right)} \lim _{t_{2} \rightarrow \infty} \sum_{s=\alpha_{2}-1}^{t_{2}-1}\left(t_{2}+\alpha_{2}-1-\sigma(s)\right)^{\alpha_{2}-1} F_{2}\left(t_{1}, s, u_{1}\left(t_{1}\right), \Delta^{\beta_{2}} u_{2}\left(s-\beta_{2}+1\right)\right) \\
& -\frac{\lambda_{1}}{\Gamma\left(\alpha_{1}\right) \Gamma\left(\theta_{1}\right)} \sum_{\xi=\alpha_{1}}^{\eta_{1}} \sum_{s=\alpha_{1}-1}^{\xi-1}\left(\eta_{1}+\theta_{1}-\sigma(\xi)\right) \frac{\theta_{1}-1}{\left(\xi+\alpha_{1}-1-\sigma(s)\right) \frac{\alpha_{1}-1}{} \times} \\
& g_{1}(s) F_{1}\left(s, t_{2}, \Delta^{\beta_{1}} u_{1}\left(s-\beta_{1}+1\right), u_{2}\left(t_{2}\right)\right) \text {. }
\end{aligned}
$$

We next make the following assumptions:

$\left(H_{1}\right)$ There exist positive numbers ${ }_{i p} \rho_{2} \in\left(-1, \rho_{2}\right)$ and $M_{i p}, m_{i p}>0(i=1,2$ and $p=1,2,3)$ such that, for each $t_{i} \in \mathbb{N}_{\alpha_{i}-2}$ and $v_{i} \in \mathbb{R}$,

$$
\begin{aligned}
\mid F_{i}\left(t_{1}, t_{2}, \frac{1}{\chi} v_{1}, \frac{1}{\chi} v_{2}\right) & -M_{i 1}\left(t_{2}+{ }_{i 1} \rho_{2}\right)^{i 1} \rho_{2} e^{-m_{i 1}\left(t_{1}+t_{2}\right)} \mid \\
\leq & M_{i 2}\left(t_{2}+{ }_{i 2} \rho_{2}\right)^{i 2} \rho_{2} e^{-m_{i 2}\left(t_{1}+t_{2}\right)}\left|v_{1}\right| \\
& +M_{i 3}\left(t_{2}+{ }_{i 3} \rho_{2}\right)^{i 3} \rho_{2} e^{-m_{i 3}\left(t_{1}+t_{2}\right)}\left|v_{2}\right| .
\end{aligned}
$$

$\left(H_{2}\right)$ There exist positive numbers ${ }_{i p} \tilde{\rho}_{i} \in\left(-1, \rho_{i}\right)$ and $N_{i p}, n_{i p}>0(i=1,2$ and $p=1,2,3)$ such that, for $v_{i} \in \mathcal{C}_{i}$

$$
\begin{aligned}
\mid \phi_{i}\left(\frac{1}{\chi} v_{1}, \frac{1}{\chi} v_{2}\right) & -N_{i 1}\left(t_{1}+{ }_{i 1} \tilde{\rho}_{1}\right)^{i 1 \tilde{\rho}_{1}}\left[\left(t_{2}+{ }_{i 1} \tilde{\rho}_{2}\right)^{i 1 \tilde{\rho}_{2}}\right]^{2} e^{-n_{i 1}\left(t_{1}+t_{2}\right)} \mid \\
\leq & N_{i 2}\left(t_{1}+{ }_{i 2} \tilde{\rho}_{1}\right)^{i 2} \tilde{\rho}_{1}\left[\left(t_{2}+{ }_{i 2} \tilde{\rho}_{2}\right)^{i 2 \tilde{\rho}_{2}}\right]^{2} e^{-n_{i 2}\left(t_{1}+t_{2}\right)}\left\|v_{1}\right\| \\
& +N_{i 3}\left(t_{1}+{ }_{i 3} \tilde{\rho}_{1}\right)^{i 3 \tilde{\rho}_{1}}\left[\left(t_{2}+{ }_{i 3} \tilde{\rho}_{2}\right)^{i 3 \tilde{\rho}_{2}}\right]^{2} e^{-n_{i 3}\left(t_{1}+t_{2}\right)}\left\|v_{2}\right\| .
\end{aligned}
$$

$\left(H_{3}\right) g_{i} \leq g_{i}\left(\eta_{i}\right)$ for all $\eta_{i} \in \mathbb{N}_{\alpha_{i}-1, T+\alpha_{i}-1}$.

Lemma 5. Suppose that $\left(H_{1}\right)-\left(H_{3}\right)$ hold. Then, the fixed point of $\mathcal{F}$ coincides with the solution of the problems (5) and (6), and $\mathcal{F}: \mathcal{U} \rightarrow \mathcal{U}$ is completely continuous.

Proof. Let $\left(u_{1}, u_{2}\right) \in \mathcal{U}$, for each $i, j \in\{1,2\}$ and $i \neq j$. By the above assumptions $\left(H_{1}\right)$ and $\left(H_{2}\right)$, it follows that:

$$
\left|F_{i}\left(t_{1}, t_{2}, \Delta^{\beta_{i}} u_{i}\left(t_{i}-\beta_{i}+1\right), u_{j}\left(t_{j}\right)\right)\right|
$$




$$
\begin{aligned}
& =\left|F_{i}\left(t_{1}, t_{2}, \frac{1}{\chi}\left[\chi \Delta^{\beta_{i}} u_{i}\left(t_{i}-\beta_{i}+1\right)\right], \frac{1}{\chi}\left[\chi u_{j}\left(t_{j}\right)\right]\right)\right| \\
& \leq M_{i 1}\left(t_{2}+{ }_{i 1} \rho_{2}\right)^{i 1} \rho_{2} e^{-m_{i 1}\left(t_{1}+t_{2}\right)}+M_{i 2}\left(t_{2}+{ }_{i 2} \rho_{2}\right)^{i 2 \rho_{2}} e^{-m_{i 2}\left(t_{1}+t_{2}\right)}\left\|\Delta^{\beta_{i}} u_{i}\right\|_{\mathcal{C}_{i}} \\
& +M_{i 3}\left(t_{2}+{ }_{i 3} \rho_{2}\right)^{i 3 \rho_{2}} e^{-m_{i 3}\left(t_{1}+t_{2}\right)}\left\|u_{j}\right\|_{\mathcal{C}_{j}} \text {, } \\
& \text { and }\left|\phi_{i}\left(u_{1}, u_{2}\right)\right|=\left|\phi_{i}\left(\frac{1}{\chi}\left[\chi u_{1}\right], \frac{1}{\chi}\left[\chi u_{2}\right]\right)\right| \\
& \leq N_{i 1}\left(t_{1}+{ }_{i 1} \tilde{\rho}_{1}\right)^{i 1} \tilde{\rho}_{1}\left[\left(t_{2}+{ }_{i 1} \tilde{\rho}_{2}\right)^{i 1 \tilde{\rho}_{2}}\right]^{2} e^{-n_{i 1}\left(t_{1}+t_{2}\right)} \\
& +N_{i 2}\left(t_{1}+{ }_{i 2} \tilde{\rho}_{1}\right)^{i 2 \tilde{\rho}_{1}}\left[\left(t_{2}+{ }_{i 2} \tilde{\rho}_{2}\right)^{i 2 \tilde{\rho}_{2}}\right]^{2} e^{-n_{i 2}\left(t_{1}+t_{2}\right)}\left\|u_{1}\right\|_{\mathcal{C}_{1}} \\
& +N_{i 3}\left(t_{1}+{ }_{i 3} \tilde{\rho}_{1}\right)^{i 3 \tilde{\rho}_{1}}\left[\left(t_{2}+{ }_{i 3} \tilde{\rho}_{2}\right)^{i 3 \tilde{\rho}_{2}}\right]^{2} e^{-n_{i 3}\left(t_{1}+t_{2}\right)}\left\|u_{2}\right\|_{\mathcal{C}_{2}} .
\end{aligned}
$$

The rest of the proof follows from Lemmas 3 and 4. This implies that the fixed point of $\mathcal{F}$ coincides with the solution of the problems (5) and (6).

To show that $\mathcal{F}$ is completely continuous, we organize the proof as the following four steps.

Step I. $\mathcal{F}$ is well defined and maps bounded sets into bounded sets.

Let $B_{R}=\left\{\left(u_{1}, u_{2}\right) \in \mathcal{U}:\left\|\left(u_{1}, u_{2}\right)\right\|_{\mathcal{U}} \leq R\right\}$, then for $\left(u_{1}, u_{2}\right) \in \mathcal{U}$ :

$$
\begin{array}{r}
R \geq \max \left\{\left\|\left(u_{1}, u_{2}\right)\right\|_{\mathcal{u}_{1}},\left\|\left(u_{1}, u_{2}\right)\right\|_{\mathcal{U}_{2}}\right\} \\
=\max \left\{\chi\left[\left|\Delta^{\beta_{1}} u_{1}\left(t_{1}-\beta_{1}+1\right)\right|+\left|u_{2}\left(t_{2}\right)\right|\right],\right. \\
\left.\chi\left[\left|u_{1}\left(t_{1}\right)\right|+\left|\Delta^{\beta_{2}} u_{2}\left(t_{2}-\beta_{2}+1\right)\right|\right]\right\} .
\end{array}
$$

By the definition of $\mathcal{F}$, we get $\mathcal{F}_{i}\left(u_{1}, u_{2}\right), \Delta^{\beta_{i}} \mathcal{F}_{i}\left(u_{1}, u_{2}\right) \in \mathcal{U}$. Therefore, (43) and (44) imply that:

$$
\begin{aligned}
& \left|F_{i}\left(t_{1}, t_{2}, \Delta^{\beta_{i}} u_{i}\left(t_{i}-\beta_{i}+1\right), u_{j}\left(t_{j}\right)\right)\right| \\
& =\left|F_{i}\left(t_{1}, t_{2}, \frac{1}{\chi}\left[\chi \Delta^{\beta_{i}} u_{i}\left(t_{i}-\beta_{i}+1\right)\right], \frac{1}{\chi}\left[\chi u_{j}\left(t_{j}\right)\right]\right)\right| \\
& \leq M_{i 1}\left(t_{2}+{ }_{i 1} \rho_{2}\right)^{i 1} \rho_{2} e^{-m_{i 1}\left(t_{1}+t_{2}\right)}+R M_{i 2}\left(t_{2}+{ }_{i 2} \rho_{2}\right)^{i 2} \rho_{2} \times \\
& e^{-m_{i 2}\left(t_{1}+t_{2}\right)}+R M_{i 3}\left(t_{2}+{ }_{i 3} \rho_{2}\right)^{i 3 \rho_{2}} e^{-m_{i 3}\left(t_{1}+t_{2}\right)}, \\
& \text { and } \\
& \left|\phi_{i}\left(u_{1}, u_{2}\right)\right|=\left|\phi_{i}\left(\frac{1}{\chi}\left[\chi u_{1}\right], \frac{1}{\chi}\left[\chi u_{2}\right]\right)\right| \\
& \leq N_{i 1}\left(t_{1}+{ }_{i 1} \tilde{\rho}_{1}\right)^{i 1 \tilde{\rho}_{1}}\left[\left(t_{2}+{ }_{i 1} \tilde{\rho}_{2}\right)^{i 1 \tilde{\rho}_{2}}\right]^{2} e^{-n_{i 1}\left(t_{1}+t_{2}\right)} \\
& +R N_{i 2}\left(t_{1}+{ }_{i 2} \tilde{\rho}_{1}\right)^{i 2 \tilde{\rho}_{1}}\left[\left(t_{2}+{ }_{i 2} \tilde{\rho}_{2}\right)^{i 2 \tilde{\rho}_{2}}\right]^{2} e^{-n_{i 2}\left(t_{1}+t_{2}\right)} \\
& +R N_{i 3}\left(t_{1}+{ }_{i 3} \tilde{\rho}_{1}\right)^{i 3 \tilde{\rho}_{1}}\left[\left(t_{2}+{ }_{i 3} \tilde{\rho}_{2}\right)^{i 3 \tilde{\rho}_{2}}\right]^{2} e^{-n_{i 3}\left(t_{1}+t_{2}\right)} .
\end{aligned}
$$

Let

$$
\begin{aligned}
\tilde{\Omega}_{1}= & :\left(N_{11}+N_{12} R+N_{13} R\right) \tilde{\Omega}_{11}+\left(N_{21}+N_{22} R+N_{23} R\right) \tilde{\Omega}_{12} \\
& +\left(M_{11}+M_{12} R+M_{13} R\right) \tilde{\Omega}_{13}+\left(M_{21}+M_{22} R+M_{23} R\right) \tilde{\Omega}_{14} \\
\tilde{\Omega}_{2}= & :\left(N_{11}+N_{12} R+N_{13} R\right) \tilde{\Omega}_{21}+\left(N_{21}+N_{22} R+N_{23} R\right) \tilde{\Omega}_{22} \\
& +\left(M_{11}+M_{12} R+M_{13} R\right) \tilde{\Omega}_{23}+\left(M_{21}+M_{22} R+M_{23} R\right) \tilde{\Omega}_{24}
\end{aligned}
$$


where:

$$
\begin{aligned}
& \tilde{\Omega}_{11}=\left[\max \left\{\frac{1}{\Gamma\left(\theta_{1}\right)}, \frac{\lambda_{1} G_{1} \Gamma\left(\alpha_{2}\right)}{\Gamma\left(\theta_{1}\right)}\left(\eta_{1}-\alpha_{1}+\theta_{1}\right) \frac{\theta_{1}-1}{} \mathcal{B}_{1}\right\}+\frac{1}{\Gamma\left(\alpha_{1}\right)}\right], \\
& \tilde{\Omega}_{12}=\max \left\{\frac{1}{\Gamma\left(\theta_{2}\right)}, \frac{\lambda_{2} G_{2} \Gamma\left(\alpha_{1}\right)}{\Gamma\left(\theta_{2}\right)}\left(\eta_{2}-\alpha_{2}+\theta_{2}\right) \frac{\theta_{2}-1}{} \mathcal{B}_{2}\right\}, \\
& \tilde{\Omega}_{13}=\left[\max \left\{1, \frac{\lambda_{1} G_{1} \Gamma\left(\alpha_{2}\right)}{\Gamma\left(\theta_{1}\right)}\left(\eta_{1}-\alpha_{1}+\theta_{1}-1\right) \frac{\theta_{1}-1}{} \mathcal{C}_{1}\right\}+\frac{1}{\Gamma\left(\alpha_{1}\right)}\right], \\
& \tilde{\Omega}_{14}=\max \left\{1, \frac{\lambda_{2} G_{2} \Gamma\left(\alpha_{1}\right)}{\Gamma\left(\theta_{2}\right)}\left(\eta_{2}-\alpha_{2}+\theta_{2}-1\right)^{\frac{\theta_{2}-1}{\mathcal{C}_{2}}}\right\},
\end{aligned}
$$

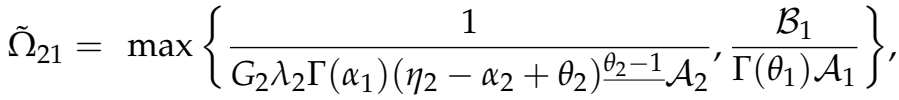

$$
\begin{aligned}
& \tilde{\Omega}_{22}=\left[\max \left\{\frac{1}{G_{1} \lambda_{1} \Gamma\left(\alpha_{2}\right)\left(\eta_{1}-\alpha_{1}+\theta_{1}\right) \frac{\theta_{1}-1}{\mathcal{A}_{1}}}, \frac{\mathcal{B}_{2}}{\Gamma\left(\theta_{2}\right) \mathcal{A}_{2}}\right\}+\frac{1}{\Gamma\left(\alpha_{2}\right)}\right] \text {, } \\
& \tilde{\Omega}_{23}=\max \left\{\frac{1}{G_{2} \lambda_{2} \Gamma\left(\alpha_{1}\right)\left(\eta_{2}-\alpha_{2}+\theta_{2}\right) \frac{\theta_{2}-1}{\mathcal{A}_{2}}}, \frac{\mathcal{C}_{1}}{\Gamma\left(\theta_{1}\right) \mathcal{A}_{1}}\right\}, \\
& \tilde{\Omega}_{24}=\left[\max \left\{\frac{1}{G_{1} \lambda_{1} \Gamma\left(\alpha_{2}\right)\left(\eta_{1}-\alpha_{1}+\theta_{1}\right) \frac{\theta_{1}-1}{\mathcal{A}_{1}},} \frac{\mathcal{C}_{2}}{\Gamma\left(\theta_{2}\right) \mathcal{A}_{2}}\right\}+\frac{1}{\Gamma\left(\alpha_{2}\right)}\right] \text {. }
\end{aligned}
$$

Hence, we obtain:

$$
\begin{aligned}
\chi & \left|\left(\mathcal{F}_{1}\left(u_{1}, u_{2}\right)\right)\left(t_{1}, t_{2}\right)\right| \\
\leq & \left(N_{11}+N_{12} R+N_{13} R\right)\left[\max \left\{\frac{1}{\Gamma\left(\theta_{1}\right)}, \frac{\lambda_{1} G_{1} \Gamma\left(\alpha_{2}\right)}{\Gamma\left(\theta_{1}\right)}\left(\eta_{1}-\alpha_{1}+\theta_{1}\right) \frac{\theta_{1}-1}{\mathcal{B}_{1}}\right\}+\frac{1}{\Gamma\left(\alpha_{1}\right)}\right] \\
& +\left(N_{21}+N_{22} R+N_{23} R\right) \max \left\{\frac{1}{\Gamma\left(\theta_{2}\right)}, \frac{\lambda_{2} G_{2} \Gamma\left(\alpha_{1}\right)}{\Gamma\left(\theta_{2}\right)}\left(\eta_{2}-\alpha_{2}+\theta_{2}\right) \underline{\theta_{2}-1} \mathcal{B}_{2}\right\} \\
& +\left(M_{11}+M_{12} R+M_{13} R\right)\left[\max \left\{1, \frac{\lambda_{1} G_{1} \Gamma\left(\alpha_{2}\right)}{\Gamma\left(\theta_{1}\right)}\left(\eta_{1}-\alpha_{1}+\theta_{1}-1\right) \frac{\theta_{1}-1}{\mathcal{C}_{1}}\right\}+\frac{1}{\Gamma\left(\alpha_{1}\right)}\right] \\
& +\left(M_{21}+M_{22} R+M_{23} R\right) \max \left\{1, \frac{\lambda_{2} G_{2} \Gamma\left(\alpha_{1}\right)}{\Gamma\left(\theta_{2}\right)}\left(\eta_{2}-\alpha_{2}+\theta_{2}-1\right) \frac{\theta_{2}-1}{\mathcal{C}_{2}}\right\} \\
= & \tilde{\Omega}_{1},
\end{aligned}
$$

and:

$$
\begin{aligned}
\chi \mid & \left(\mathcal{F}_{2}\left(u_{1}, u_{2}\right)\right)\left(t_{1}, t_{2}\right) \mid \\
\leq & \left(N_{11}+N_{12} R+N_{13} R\right) \max \left\{\frac{1}{G_{2} \lambda_{2} \Gamma\left(\alpha_{1}\right)\left(\eta_{2}-\alpha_{2}+\theta_{2}\right) \frac{\theta_{2}-1}{\mathcal{A}_{2}}}, \frac{\mathcal{B}_{1}}{\Gamma\left(\theta_{1}\right) \mathcal{A}_{1}}\right\} \\
+ & \left(N_{21}+N_{22} R+N_{23} R\right) \times \\
& {\left[\max \left\{\frac{1}{G_{1} \lambda_{1} \Gamma\left(\alpha_{2}\right)\left(\eta_{1}-\alpha_{1}+\theta_{1}\right) \frac{\theta_{1}-1}{\mathcal{A}_{1}}}, \frac{\mathcal{B}_{2}}{\Gamma\left(\theta_{2}\right) \mathcal{A}_{2}}\right\}+\frac{1}{\Gamma\left(\alpha_{2}\right)}\right] } \\
+ & \left(M_{11}+M_{12} R+M_{13} R\right) \max \left\{\frac{1}{G_{2} \lambda_{2} \Gamma\left(\alpha_{1}\right)\left(\eta_{2}-\alpha_{2}+\theta_{2}\right) \frac{\theta_{2}-1}{\mathcal{A}_{2}}}, \frac{\mathcal{C}_{1}}{\Gamma\left(\theta_{1}\right) \mathcal{A}_{1}}\right\} \\
+ & \left(M_{21}+M_{22} R+M_{23} R\right) \times \\
& {\left[\operatorname { m a x } \left\{\frac{1}{\left.\left.G_{1} \lambda_{1} \Gamma\left(\alpha_{2}\right)\left(\eta_{1}-\alpha_{1}+\theta_{1}\right) \frac{\theta_{1}-1}{\mathcal{A}_{1}}, \frac{\mathcal{C}_{2}}{\Gamma\left(\theta_{2}\right) \mathcal{A}_{2}}\right\}+\frac{1}{\Gamma\left(\alpha_{2}\right)}\right]}\right.\right.}
\end{aligned}
$$




$$
=\tilde{\Omega}_{2} .
$$

Similarly, we have:

$$
\begin{aligned}
& \chi\left|\Delta^{\beta_{1}}\left(\mathcal{F}_{1}\left(u_{1}, u_{2}\right)\right)\left(t_{1}-\beta_{1}+1, t_{2}\right)\right|<\tilde{\Omega}_{1}, \\
& \chi\left|\Delta^{\beta_{2}}\left(\mathcal{F}_{2}\left(u_{1}, u_{2}\right)\right)\left(t_{1}, t_{2}-\beta_{2}+1\right)\right|<\tilde{\Omega}_{2} .
\end{aligned}
$$

Therefore, $\mathcal{F}_{i}\left(u_{1}, u_{2}\right) \in \mathcal{U}$. This implies that $\mathcal{F}: \mathcal{U} \longrightarrow \mathcal{U}$ is well defined.

Furthermore, we obtain:

$$
\begin{aligned}
\left\|\mathcal{F}\left(u_{1}, u_{2}\right)\right\|_{\mathcal{U}_{i}} & =\max \left\{\chi\left|\Delta^{\beta_{i}}\left(\mathcal{F}_{i}\left(u_{1}, u_{2}\right)\right)\left(t_{i}-\beta_{i}+1, t_{j}\right)\right|\right. \\
& \left.+\chi\left|\left(\mathcal{F}_{j}\left(u_{1}, u_{2}\right)\right)\left(t_{i}, t_{j}\right)\right| \text { for } i, j \in\{1,2\}, i \neq j\right\} .
\end{aligned}
$$

Hence,

$$
\left\|\mathcal{F}\left(u_{1}, u_{2}\right)\right\|_{\mathcal{U}}=\max \left\{\left\|\mathcal{F}\left(u_{1}, u_{2}\right)\right\|_{\mathcal{U}_{1}},\left\|\mathcal{F}\left(u_{1}, u_{2}\right)\right\|_{\mathcal{U}_{2}}\right\}<\tilde{\Omega}_{1}+\tilde{\Omega}_{2}
$$

Thus, $\mathcal{F}$ maps bounded sets into bounded sets.

Step II. $\mathcal{F}$ is continuous.

Let $\epsilon>0$ be given. Since $F_{i}$ and $\phi_{i}$ are continuous, then $F_{i}$ and $\phi_{i}$ are uniformly continuous. Therefore, there exists $\delta=\min \left\{\delta_{i}, \hat{\delta}_{i}\right\}>0$ such that, for each $t_{i} \in \mathcal{N}_{\alpha_{i}-2}, u_{i}, v_{i} \in \mathcal{C}_{i}$ with $\max \left\{\chi\left|\Delta^{\beta_{i}} u_{i}\left(t_{i}-\beta_{i}+1\right)-\Delta^{\beta_{i}} v_{i}\left(t_{i}\right)\right|+\chi\left|u_{j}\left(t_{i}\right)-v_{j}\left(t_{j}\right)\right|\right\}<\delta_{i}$

$$
\begin{aligned}
& \left|F_{i}\left(t_{1}, t_{2}, \Delta^{\beta_{i}} u_{i}\left(t_{i}-\beta_{i}+1\right), u_{j}\left(t_{j}\right)\right)-F_{i}\left(t_{1}, t_{2}, \Delta^{\beta_{i}} v_{i}\left(t_{i}-\beta_{i}+1\right), v_{j}\left(t_{j}\right)\right)\right| \\
= & \mid F_{i}\left(t_{1}, t_{2}, \frac{1}{\chi}\left[\chi \Delta^{\beta_{i}} u_{i}\left(t_{i}-\beta_{i}+1\right)\right], \frac{1}{\chi}\left[\chi u_{j}\left(t_{j}\right)\right]\right) \\
& -F_{i}\left(t_{1}, t_{2}, \frac{1}{\chi}\left[\chi \Delta^{\beta_{i}} v_{i}\left(t_{i}-\beta_{i}+1\right)\right], \frac{1}{\chi}\left[\chi v_{j}\left(t_{j}\right)\right]\right) \mid \\
< & 2 M_{i 1}+2 M_{i 2} R+2 M_{i 3} R<\frac{\epsilon}{4 \Omega_{i}} .
\end{aligned}
$$

For each $u_{i}, v_{i} \in \mathcal{C}_{i}$ with $\left|u_{i}-v_{i}\right|<\hat{\delta}_{i}$

$$
\begin{aligned}
\left|\phi_{i}\left(u_{1}, u_{2}\right)-\phi_{i}\left(v_{1}, v_{2}\right)\right| & =\left|\phi_{i}\left(\frac{1}{\chi}\left[\chi u_{1}\right], \frac{1}{\chi}\left[\chi u_{2}\right]\right)-\phi_{i}\left(\frac{1}{\chi}\left[\chi v_{1}\right], \frac{1}{\chi}\left[\chi v_{2}\right]\right)\right| \\
& <2 N_{i 1}+2 N_{i 2} R+2 N_{i 3} R<\frac{\epsilon}{4 \Omega_{i}} .
\end{aligned}
$$

Similar to Step I, we obtain:

$$
\chi\left|\left(\mathcal{F}_{i}\left(u_{1}, u_{2}\right)\right)-\left(\mathcal{F}_{i}\left(v_{1}, v_{2}\right)\right)\right|<2 \tilde{\Omega}_{i}<\frac{\epsilon}{2}
$$

and

$$
\chi\left|\Delta^{\beta_{i}}\left(\mathcal{F}_{i}\left(u_{1}, u_{2}\right)\right)-\Delta^{\beta_{i}}\left(\mathcal{F}_{i}\left(v_{1}, v_{2}\right)\right)\right|<2 \tilde{\Omega}_{i}<\frac{\epsilon}{2} .
$$

Thus, we have:

$$
\begin{aligned}
& \left\|\mathcal{F}_{i}\left(u_{1}, u_{2}\right)-\mathcal{F}_{i}\left(v_{1}, v_{2}\right)\right\|_{\mathcal{U}_{i}} \\
= & \left\|\Delta^{\beta_{i}} \mathcal{F}_{i}\left(u_{1}, u_{2}\right)-\Delta^{\beta_{i}} \mathcal{F}_{i}\left(v_{1}, v_{2}\right)\right\|_{\mathcal{C}_{i}}+\left\|\mathcal{F}_{j}\left(u_{1}, u_{2}\right)-\mathcal{F}_{j}\left(v_{1}, v_{2}\right)\right\|_{\mathcal{C}_{j}} \\
< & 2\left(\tilde{\Omega}_{1}+\tilde{\Omega}_{2}\right)<\epsilon .
\end{aligned}
$$


This means that each $\mathcal{F}_{i}, i=1,2$ is continuous. This shows $\mathcal{F}$ is continuous.

In order to prove that $\mathcal{F}$ maps bounded sets of $\mathcal{U} \subset \mathcal{C}_{1} \times \mathcal{C}_{2}$ to relatively compact sets of $\mathcal{U} \subset \mathcal{C}_{1} \times \mathcal{C}_{2}$, it suffices to show that both $\mathcal{F}_{1}$ and $\mathcal{F}_{2}$ map bounded sets to relatively compact sets. Let $\Theta_{i} \subset \mathcal{C}_{i}, i=1,2$ be bounded sets and $\Theta_{1} \times \Theta_{2} \subset \mathcal{U}$. Recall that $\Theta_{i}$ are relatively compact if:

- both $\Theta_{i}$ are bounded,

- both $\chi \Theta_{i}$ are equicontinuous on any closed subintervals of $\mathbb{N}_{\alpha_{i}-2}$,

- $\quad$ both $\chi \Theta_{i}$ are equiconvergent as $t_{i} \rightarrow \infty$.

It has been shown from in Step I that both $\mathcal{F}_{i}$ are uniformly bounded. Now, we show that $\mathcal{F}_{i}$ maps bounded sets into equicontinuous sets of $\mathcal{U}$.

Step III. Both $\mathcal{F}_{i}: \Theta_{1} \times \Theta_{2} \rightarrow \mathcal{U}$ are equicontinuous on $\left(\left[a_{1}, b_{1}\right] \cap \mathbb{N}_{\alpha_{1}-2}\right) \times\left(\left[a_{2}, b_{2}\right] \cap \mathbb{N}_{\alpha_{2}-2}\right):=\mathcal{D}$.

For any $\epsilon>0$, there exists $\delta>0$ such that, for each $t_{i 1}, t_{i 2} \in \mathbb{N}_{\alpha_{i}-2} \cap\left[a_{i}, b_{i}\right]$,

$$
\left|\left(t_{11}+\rho_{1}\right)^{\underline{\rho}_{1}}\left(t_{21}+\rho_{2}\right)^{\underline{\rho}_{2}}-\left(t_{12}+\rho_{1}\right)^{\underline{\rho}_{1}}\left(t_{22}+\rho_{2}\right)^{\underline{\rho}_{2}}\right| \leq \frac{\epsilon}{2 \max \left\{\tilde{\Omega}_{1}^{*}+\tilde{\Omega}_{2}^{*}\right\}}=\delta,
$$

where:

$$
\begin{aligned}
\tilde{\Omega}_{1}^{*}= & \left(\left[N_{11}+N_{12} R+N_{13} R\right]+\left[M_{11}+M_{12} R+M_{13} R\right]\right)\left[\frac{1}{\Gamma\left(\theta_{1}\right)}+\frac{1}{\Gamma\left(\alpha_{1}\right)}\right] \\
+ & \left(\left[N_{11}+N_{12} R+N_{13} R\right] \mathcal{B}_{1}+\left[M_{11}+M_{12} R+M_{13} R\right] \mathcal{C}_{1}\right) \times \\
& \frac{\lambda_{1} G_{1} \Gamma\left(\alpha_{2}\right)\left(\eta_{1}-\alpha_{1}+\theta_{1}\right) \frac{\theta_{1}-1}{\Gamma\left(\theta_{1}\right) \Gamma\left(\theta_{2}\right)}}{+} \\
+ & \left(\left[N_{21}+N_{22} R+N_{23} R\right]+\left[M_{21}+M_{22} R+M_{23} R\right]\right) \frac{1}{\Gamma\left(\theta_{2}\right)} \\
+ & \left(\left[N_{21}+N_{22} R+N_{23} R\right] \mathcal{B}_{2}+\left[M_{21}+M_{22} R+M_{23} R\right] \mathcal{C}_{2}\right) \times \\
& \left.\frac{\lambda_{2} G_{2} \Gamma\left(\alpha_{1}\right)\left(\eta_{2}-\alpha_{2}+\theta_{2}\right) \frac{\theta_{2}-1}{\Gamma\left(\theta_{1}\right) \Gamma\left(\theta_{2}\right)},}{\tilde{\Omega}_{2}^{*}=\quad}, N_{11}+N_{12} R+N_{13} R\right]\left[\frac{1}{\lambda_{2} G_{2}\left(\eta_{2}-\alpha_{2}+\theta_{2}\right) \frac{\theta_{2}-1}{\mathcal{A}_{2}}}+\frac{1}{\Gamma\left(\alpha_{2}\right)}\right] \\
+ & {\left[N_{21}+N_{22} R+N_{23} R\right] \frac{1}{\lambda_{1} G_{1}\left(\eta_{1}-\alpha_{1}+\theta_{1}\right) \frac{\theta_{1}-1}{\mathcal{A}_{1}}} } \\
+ & {\left[M_{11}+M_{12} R+M_{13} R\right] \frac{\mathcal{B}_{1}}{\Gamma\left(\theta_{1}\right) \mathcal{A}_{1}}+\left[M_{21}+M_{22} R+M_{23} R\right] \frac{\mathcal{B}_{2}}{\Gamma\left(\theta_{2}\right) \mathcal{A}_{2}} . }
\end{aligned}
$$

Hence, for each $t_{i 1}, t_{i 2} \in \mathbb{N}_{\alpha_{i}-2} \cap\left[a_{i}, b_{i}\right]$, and $u_{i} \in \Theta_{i}$, we have:

$$
\begin{aligned}
& \left|\chi\left(\mathcal{F}_{1} u_{1}\right)\left(t_{11}, t_{21}\right)-\chi\left(\mathcal{F}_{1} u_{1}\right)\left(t_{12}, t_{22}\right)\right| \\
& \leq \chi \mid t_{11}^{\alpha_{1}}\left\{\left[\frac{\left|\phi_{1}\left(u_{1}, u_{2}\right)\right|}{\Gamma\left(\theta_{1}\right)}-\frac{\left|\phi_{2}\left(u_{1}, u_{2}\right)\right|}{\Gamma\left(\theta_{2}\right)}+\frac{\left|\phi_{1}\left(u_{1}, u_{2}\right)\right|}{\Gamma\left(\alpha_{1}\right)}\right]+\frac{1}{\Gamma\left(\theta_{1}\right) \Gamma\left(\theta_{2}\right)} \times\right. \\
& {\left[\frac{\lambda_{1} \Gamma\left(\alpha_{2}\right) \mathcal{B}_{1}\left|\phi_{1}\left(u_{1}, u_{2}\right)\right|}{t_{21}^{\frac{\alpha_{2}-1}{21}} \Gamma\left(\alpha_{1}\right)}+\frac{\lambda_{2} \Gamma\left(\alpha_{1}\right) \mathcal{B}_{2}\left|\phi_{2}\left(u_{1}, u_{2}\right)\right|}{t_{11}^{\frac{\alpha_{1}-1}{11}} \Gamma\left(\alpha_{2}\right)}\right]+\frac{1}{\Gamma\left(\theta_{1}\right) \Gamma\left(\theta_{2}\right)} \times} \\
& +\left[\frac{\lambda_{1} \Gamma\left(\alpha_{2}\right) \mathcal{B}_{1}\left|F_{1}\left(t_{1}, t_{2}, \Delta^{\beta_{1}} u_{1}, u_{2}\right)\right|}{t_{\frac{\alpha_{2}}{22}} \Gamma\left(\alpha_{1}\right)}+\frac{\lambda_{2} \Gamma\left(\alpha_{1}\right) \mathcal{B}_{2}\left|F_{2}\left(t_{1}, t_{2}, u_{1}, \Delta^{\beta_{2}} u_{2}\right)\right|}{t^{\frac{\alpha_{1}-1}{12}} \Gamma\left(\alpha_{2}\right)}\right] \\
& \left.\left[\frac{\left|F_{1}\left(t_{1}, t_{2}, \Delta^{\beta_{1}} u_{1}, u_{2}\right)\right|}{\Gamma\left(\theta_{1}\right)}-\frac{\left|F_{2}\left(t_{1}, t_{2}, u_{1}, \Delta^{\beta_{2}} u_{2}\right)\right|}{\Gamma\left(\theta_{2}\right)}+\frac{\left|F_{1}\left(t_{1}, t_{2}, \Delta^{\beta_{1}} u_{1}, u_{2}\right)\right|}{\Gamma\left(\alpha_{1}\right)}\right]\right\}
\end{aligned}
$$




$$
\begin{aligned}
& -t \frac{\alpha_{1}}{12}\left\{\left[\frac{\left|\phi_{1}\left(u_{1}, u_{2}\right)\right|}{\Gamma\left(\theta_{1}\right)}-\frac{\left|\phi_{2}\left(u_{1}, u_{2}\right)\right|}{\Gamma\left(\theta_{2}\right)}+\frac{\left|\phi_{1}\left(u_{1}, u_{2}\right)\right|}{\Gamma\left(\alpha_{1}\right)}\right]+\frac{1}{\Gamma\left(\theta_{1}\right) \Gamma\left(\theta_{2}\right)} \times\right. \\
& {\left[\frac{\lambda_{1} \Gamma\left(\alpha_{2}\right) \mathcal{B}_{1}\left|\phi_{1}\left(u_{1}, u_{2}\right)\right|}{t_{21}^{\alpha_{2}-1} \Gamma\left(\alpha_{1}\right)}+\frac{\lambda_{2} \Gamma\left(\alpha_{1}\right) \mathcal{B}_{2}\left|\phi_{2}\left(u_{1}, u_{2}\right)\right|}{t_{11}^{\alpha_{1}-1} \Gamma\left(\alpha_{2}\right)}\right]+\frac{1}{\Gamma\left(\theta_{1}\right) \Gamma\left(\theta_{2}\right)} \times} \\
& +\left[\frac{\lambda_{1} \Gamma\left(\alpha_{2}\right) \mathcal{B}_{1}\left|F_{1}\left(t_{1}, t_{2}, \Delta^{\beta_{1}} u_{1}, u_{2}\right)\right|}{t_{22}^{\alpha_{2}-1} \Gamma\left(\alpha_{1}\right)}+\frac{\lambda_{2} \Gamma\left(\alpha_{1}\right) \mathcal{B}_{2}\left|F_{2}\left(t_{1}, t_{2}, u_{1}, \Delta^{\beta_{2}} u_{2}\right)\right|}{t_{12}^{\frac{\alpha_{1}-1}{12}} \Gamma\left(\alpha_{2}\right)}\right] \\
& \left.\left[\frac{\left|F_{1}\left(t_{1}, t_{2}, \Delta^{\beta_{1}} u_{1}, u_{2}\right)\right|}{\Gamma\left(\theta_{1}\right)}-\frac{\left|F_{2}\left(t_{1}, t_{2}, u_{1}, \Delta^{\beta_{2}} u_{2}\right)\right|}{\Gamma\left(\theta_{2}\right)}+\frac{\left|F_{1}\left(t_{1}, t_{2}, \Delta^{\beta_{1}} u_{1}, u_{2}\right)\right|}{\Gamma\left(\alpha_{1}\right)}\right]\right\} \mid \\
& <\left|\left(t_{11}+\rho_{1}\right)^{\rho_{1}}\left(t_{21}+\rho_{2}\right)^{\underline{\rho}_{2}}-\left(t_{12}+\rho_{1}\right)^{\rho_{1}}\left(t_{22}+\rho_{2}\right) \underline{\rho_{2}}\right| \tilde{\Omega}_{1}^{*} \\
& <\frac{\epsilon}{2}
\end{aligned}
$$

and:

$$
\begin{aligned}
& \left|\chi\left(\mathcal{F}_{2} u_{2}\right)\left(t_{11}, t_{21}\right)-\chi\left(\mathcal{F}_{2} u_{2}\right)\left(t_{12}, t_{22}\right)\right| \\
& \leq \chi \mid t_{21}^{\alpha_{2}}\left\{\left[\frac{t \frac{\alpha_{1}-1}{11}\left|\phi_{1}\left(u_{1}, u_{2}\right)\right|}{\lambda_{2} G_{2}\left(\eta_{2}-\alpha_{2}+\theta_{2}\right) \frac{\theta_{2}-1}{\mathcal{A}_{2}}}-\frac{t \frac{\alpha_{2}-1}{21}\left|\phi_{2}\left(u_{1}, u_{2}\right)\right|}{\lambda_{1} G_{1}\left(\eta_{1}-\alpha_{1}+\theta_{1}\right) \frac{\theta_{1}-1}{\mathcal{A}_{1}}}+\frac{\left|\phi_{2}\left(u_{1}, u_{2}\right)\right|}{\Gamma\left(\alpha_{2}\right)}\right]\right. \\
& +t_{21}^{\alpha_{2}-1}\left[\frac{\mathcal{B}_{1}\left|\phi_{1}\left(u_{1}, u_{2}\right)\right|}{\Gamma\left(\theta_{1}\right) \mathcal{A}_{1}}-\frac{\mathcal{B}_{2}\left|\phi_{2}\left(u_{1}, u_{2}\right)\right|}{\Gamma\left(\theta_{2}\right) \mathcal{A}_{2}}\right]+\left[\frac{t \frac{\alpha_{1}-1}{11}\left|F_{1}\left(t_{1}, t_{2}, \Delta \Delta^{\beta_{1}} u_{1}, u_{2}\right)\right|}{\lambda_{2} G_{2}\left(\eta_{2}-\alpha_{2}+\theta_{2}\right) \underline{\theta_{2}-1} \mathcal{A}_{2}}\right. \\
& \left.-\frac{t_{21}^{\alpha_{2}-1}\left|F_{2}\left(t_{1}, t_{2}, u_{1}, \Delta^{\beta_{2}} u_{2}\right)\right|}{\lambda_{1} G_{1}\left(\eta_{1}-\alpha_{1}+\theta_{1}\right) \frac{\theta_{1}-1}{-} \mathcal{A}_{1}}+\frac{\left|F_{2}\left(t_{1}, t_{2}, u_{1}, \Delta^{\beta_{2}} u_{2}\right)\right|}{\Gamma\left(\alpha_{2}\right)}\right] \\
& \left.+t_{21}^{\alpha_{2}-1}\left[\frac{t_{11}^{\alpha_{1}} \mathcal{C}_{1}\left|F_{1}\left(t_{1}, t_{2}, \Delta^{\beta_{1}} u_{1}, u_{2}\right)\right|}{\Gamma\left(\theta_{1}\right) \mathcal{A}_{1}}-\frac{t_{21}^{\alpha_{2}} \mathcal{C}_{2}\left|F_{2}\left(t_{1}, t_{2}, u_{1}, \Delta^{\beta_{2}} u_{2}\right)\right|}{\Gamma\left(\theta_{2}\right) \mathcal{A}_{2}}\right]\right\} \\
& -t_{22}^{\alpha_{2}}\left\{\left[\frac{t_{11}^{\alpha_{1}-1}\left|\phi_{1}\left(u_{1}, u_{2}\right)\right|}{\lambda_{2} G_{2}\left(\eta_{2}-\alpha_{2}+\theta_{2}\right) \frac{\theta_{2}-1}{2} \mathcal{A}_{2}}-\frac{t_{21}^{\alpha_{2}-1}}{\lambda_{1} G_{1}\left(\eta_{1}-\alpha_{1}+\theta_{1}\right) \frac{\theta_{1}-1}{\mathcal{A}_{1}}}+\frac{\left|u_{2}\left(u_{1}, u_{2}\right)\right|}{\Gamma\left(\alpha_{2}\right)}\right]\right. \\
& +t_{21}^{\alpha_{2}-1}\left[\frac{\mathcal{B}_{1}\left|\phi_{1}\left(u_{1}, u_{2}\right)\right|}{\Gamma\left(\theta_{1}\right) \mathcal{A}_{1}}-\frac{\mathcal{B}_{2}\left|\phi_{2}\left(u_{1}, u_{2}\right)\right|}{\Gamma\left(\theta_{2}\right) \mathcal{A}_{2}}\right]+\left[\frac{t \frac{\alpha_{1}-1}{11}\left|F_{1}\left(t_{1}, t_{2}, \Delta^{\beta_{1}} u_{1}, u_{2}\right)\right|}{\lambda_{2} G_{2}\left(\eta_{2}-\alpha_{2}+\theta_{2}\right) \frac{\theta_{2}-1}{\mathcal{A}_{2}}}\right. \\
& \left.-\frac{t_{21}^{\alpha_{2}-1}\left|F_{2}\left(t_{1}, t_{2}, u_{1}, \Delta^{\beta_{2}} u_{2}\right)\right|}{\lambda_{1} G_{1}\left(\eta_{1}-\alpha_{1}+\theta_{1}\right) \frac{\theta_{1}-1}{\mathcal{A}_{1}}}+\frac{\left|F_{2}\left(t_{1}, t_{2}, u_{1}, \Delta^{\beta_{2}} u_{2}\right)\right|}{\Gamma\left(\alpha_{2}\right)}\right] \\
& \left.+t_{21}^{\alpha_{2}-1}\left[\frac{t_{11}^{\frac{\alpha_{1}}{11}} \mathcal{C}_{1}\left|F_{1}\left(t_{1}, t_{2}, \Delta^{\beta_{1}} u_{1}, u_{2}\right)\right|}{\Gamma\left(\theta_{1}\right) \mathcal{A}_{1}}-\frac{t_{21}^{\alpha_{2}} \mathcal{C}_{2}\left|F_{2}\left(t_{1}, t_{2}, u_{1}, \Delta^{\beta_{2}} u_{2}\right)\right|}{\Gamma\left(\theta_{2}\right) \mathcal{A}_{2}}\right]\right\} \\
& <\left|\left(t_{11}+\rho_{1}\right)^{\underline{\rho_{1}}}\left(t_{21}+\rho_{2}\right)^{\underline{\rho_{2}}}-\left(t_{12}+\rho_{1}\right)^{\underline{\rho_{1}}}\left(t_{22}+\rho_{2}\right)^{\underline{\rho_{2}}}\right| \tilde{\Omega}_{2}^{*} \\
& <\frac{\epsilon}{2} \text {. }
\end{aligned}
$$

Similarly, for each $i, j \in\{1,2\}$ and $j \neq i$, we obtain:

$$
\left|\Delta^{\beta_{i}}\left(\mathcal{F}_{i}\left(u_{1}, u_{2}\right)\right)\left(t_{i 1}-\beta_{i}+1, t_{j 1}\right)-\Delta^{\beta_{i}}\left(\mathcal{F}_{i}\left(u_{1}, u_{2}\right)\right)\left(t_{i 2}-\beta_{i}+1, t_{j 2}\right)\right|<\frac{\epsilon}{2} .
$$

Hence:

$$
\begin{aligned}
& \left\|\mathcal{F}_{i}\left(u_{1}, u_{2}\right)\left(t_{11}, t_{21}\right)-\mathcal{F}_{i}\left(u_{1}, u_{2}\right)\left(t_{12}, t_{22}\right)\right\|_{\mathcal{U}_{i}} \\
= & \left\|\Delta^{\beta_{i}} \mathcal{F}_{i}\left(u_{1}, u_{2}\right)\left(t_{i 1}-\beta_{i}+1, t_{j 1}\right)-\Delta^{\beta_{i}} \mathcal{F}_{i}\left(u_{1}, u_{2}\right)\left(t_{i 2}-\beta_{i}+1, t_{j 2}\right)\right\|_{\mathcal{C}_{i}}
\end{aligned}
$$




$$
\begin{aligned}
& +\left\|\mathcal{F}_{j}\left(u_{1}, u_{2}\right)\left(t_{11}, t_{21}\right)-\mathcal{F}_{j}\left(u_{1}, u_{2}\right)\left(t_{12}, t_{22}\right)\right\|_{\mathcal{C}_{j}} \\
& <\frac{\epsilon}{2}+\frac{\epsilon}{2}=\epsilon .
\end{aligned}
$$

This implies that both $\mathcal{F}_{1}$ and $\mathcal{F}_{2}$ are equicontinuous on $\mathcal{D}$, which shows that $\mathcal{F}$ is equicontinuous on $\mathcal{D}$. Therefore, by the Arzelá-Ascoli theorem and Theorem 2, we can conclude that $\mathcal{F}$ is completely continuous.

Step IV. Both $\mathcal{F}_{i}: \Theta_{1} \times \Theta_{2} \rightarrow \mathcal{U}$ are equiconvergent as $t_{1}, t_{2} \rightarrow \infty$.

By the assumption $(H 1)-(H 2)$, we obtain:

$$
\begin{aligned}
& \chi\left|\left(\mathcal{F}_{1}\left(u_{1}, u_{2}\right)\right)\left(t_{1}, t_{2}\right)\right|<\frac{\tilde{\Omega}_{1}^{*}}{t_{1}^{\frac{\alpha_{1}}{1}} t \frac{\alpha_{2}}{2}} \rightarrow 0 \text { uniformly in } \Theta_{1} \times \Theta_{2} \text { as } t_{1}, t_{2} \rightarrow \infty, \\
& \chi\left|\left(\mathcal{F}_{2}\left(u_{1}, u_{2}\right)\right)\left(t_{1}, t_{2}\right)\right|<\frac{\tilde{\Omega}_{2}^{*}}{t_{1}^{\frac{\alpha_{1}}{1}} t_{2}^{\frac{\alpha_{2}}{2}}} \rightarrow 0 \text { uniformly in } \Theta_{1} \times \Theta_{2} \text { as } t_{1}, t_{2} \rightarrow \infty,
\end{aligned}
$$

where $\tilde{\Omega}_{1}^{*}, \tilde{\Omega}_{2}^{*}$ are defined as (58) and (59).

Furthermore, we have:

$$
\begin{aligned}
\chi\left|\Delta^{\beta_{1}}\left(\mathcal{F}_{1}\left(u_{1}, u_{2}\right)\right)\left(t_{1}-\beta_{1}+1, t_{2}\right)\right|< & \frac{\tilde{\Omega}_{1}^{*}}{t_{1}^{\alpha_{1}} t_{2}^{\alpha_{2}}} \rightarrow 0 \\
& \text { uniformly in } \Theta_{1} \times \Theta_{2} \text { as } t_{1}, t_{2} \rightarrow \infty, \\
\chi\left|\Delta^{\beta_{2}}\left(\mathcal{F}_{2}\left(u_{1}, u_{2}\right)\right)\left(t_{1}, t_{2}-\beta_{2}+1\right)\right|< & \frac{\tilde{\Omega}_{2}^{*}}{t_{1}^{\alpha_{1}} t_{2}^{\alpha_{2}}} \rightarrow 0 \\
& \text { uniformly in } \Theta_{1} \times \Theta_{2} \text { as } t_{1}, t_{2} \rightarrow \infty .
\end{aligned}
$$

Hence, both $\mathcal{F}_{i}$ are equiconvergent as $t_{1}, t_{2} \rightarrow \infty$.

Consequently, from Step I-Step IV, we conclude that $\mathcal{F}$ is completely continuous. This complete the proof.

Finally, we present the main result of the article. For the sake of convenience, we set:

$$
\begin{aligned}
& \text { and } \begin{aligned}
\Psi_{1}= & \left(N_{12}+N_{13}\right) \tilde{\Omega}_{11}+\left(N_{22}+N_{23}\right) \tilde{\Omega}_{12}+\left(M_{12}+M_{13}\right) \tilde{\Omega}_{13} \\
& +\left(M_{22}+M_{23}\right) \tilde{\Omega}_{14}, \\
\Psi_{2}= & \left(N_{12}+N_{13}\right) \tilde{\Omega}_{21}+\left(N_{22}+N_{23}\right) \tilde{\Omega}_{22}+\left(M_{12}+M_{13}\right) \tilde{\Omega}_{23} \\
& +\left(M_{22}+M_{23}\right) \tilde{\Omega}_{24},
\end{aligned}
\end{aligned}
$$

where $\tilde{\Omega}_{1 p}, \tilde{\Omega}_{2 p}, p=1,2,3,4$ are defined as (48) and (49).

Theorem 4. Suppose that $\left(H_{1}\right)-\left(H_{2}\right)$ hold. Then, the problems (5) and (6) has at least one solution if:

$$
\Psi_{1}+\Psi_{2}<1
$$

Proof. Under the Banach space $\mathcal{U}$ equipped with the norm $\|\cdot\|_{\mathcal{U}}$, we let:

$$
\begin{aligned}
& \omega_{1}\left(t_{1}, t_{2}\right) \\
= & \frac{t_{1}^{\alpha_{1}-1}}{\Lambda}\left\{\frac { \lambda _ { 1 } G _ { 1 } \Gamma ( \alpha _ { 1 } ) ( \eta _ { 1 } - \alpha _ { 1 } + \theta _ { 1 } ) \underline { \theta _ { 1 } - 1 } \mathcal { A } _ { 1 } } { \Gamma ( \theta _ { 1 } ) } \left[\left(t_{1}+\rho_{1}\right) \underline{\rho}^{\rho_{1}} e^{-n_{1}\left(t_{1}+1\right)} \times\right.\right.
\end{aligned}
$$




$$
\begin{aligned}
& \left(\frac{N_{11} t \frac{\alpha_{1}-2}{1}}{\Gamma\left(\alpha_{1}\right)}-\frac{N_{21} \lambda_{2} G_{2}\left(\eta_{2}-\alpha_{2}+\theta_{2}\right) \frac{\theta_{2}-1}{B_{2}}}{\Gamma\left(\theta_{2}\right)}\right)+\frac{M_{11} e^{-m_{1} t_{2}}}{\Gamma\left(\alpha_{1}\right)} \times \\
& \left.\sum_{s=0}^{t_{1}-\alpha_{1}}\left(t_{1}-\sigma(s)\right) \frac{\alpha_{1}-1}{2} e^{-2 m_{1} s}-\frac{M_{21} G_{2}\left(\eta_{2}-\alpha_{2}+\theta_{2}-1\right) \underline{\theta_{2}-1} e^{-m_{1}\left(2 t_{1}+t_{2}\right)}}{\Gamma\left(\theta_{2}\right)}\right] \\
& +\frac{\lambda_{2} G_{2} \Gamma\left(\alpha_{2}\right)\left(\eta_{2}-\alpha_{2}+\theta_{2}\right) \underline{\theta_{2}-1} \mathcal{A}_{2}}{\Gamma\left(\theta_{2}\right)}\left[\left(t_{1}+\rho_{1}\right) \underline{\rho_{1}} e^{-n_{1}\left(t_{1}+1\right)} \times\right. \\
& \left(\frac{N_{21} t \frac{\alpha_{2}-2}{2}}{\Gamma\left(\alpha_{2}\right)}-\frac{N_{11} \lambda_{1} G_{1}\left(\eta_{1}-\alpha_{1}+\theta_{1}\right) \frac{\theta_{1}-1}{\mathcal{B}_{1}}}{\Gamma\left(\theta_{1}\right)}\right)+\frac{M_{21} e^{-2 m_{1} t_{1}}}{\Gamma\left(\alpha_{2}\right)} \times \\
& \left.\left.\sum_{s=0}^{t_{2}-\alpha_{2}}\left(t_{2}-\sigma(s)\right) \frac{\alpha_{2}-1}{2} e^{-m_{1} s}-\frac{M_{11} G_{1}\left(\eta_{1}-\alpha_{1}+\theta_{1}-1\right) \frac{\theta_{1}-1}{2} e^{-m_{1}\left(2 t_{1}+t_{2}\right)}}{\Gamma\left(\theta_{1}\right)}\right]\right\} \\
& +\frac{N_{11}}{\Gamma\left(\alpha_{1}\right)}\left(t_{1}+\rho_{1}\right) \frac{\rho_{1}}{2} e^{-n_{1}\left(t_{1}+1\right)}+\frac{M_{11} e^{-m_{1} t_{2}}}{\Gamma\left(\alpha_{1}\right)} \sum_{s=0}^{t_{1}-\alpha_{1}}\left(t_{1}-\sigma(s)\right) \frac{\alpha_{1}-1}{1} e^{-2 m_{1} s},
\end{aligned}
$$

and

$$
\begin{aligned}
& \omega_{2}\left(t_{1}, t_{2}\right) \\
& =\frac{t_{\frac{\alpha_{2}-1}{2}}}{\Lambda}\left\{t ^ { \frac { \alpha _ { 2 } - 1 } { 2 } } \left[( t _ { 1 } + \rho _ { 1 } ) ^ { \frac { \rho _ { 1 } } { 1 } } [ ( t _ { 2 } + \rho _ { 2 } ) ^ { \frac { \rho _ { 2 } } { 2 } } ] ^ { 2 } e ^ { - n _ { 2 } ( t _ { 2 } + 1 ) } \left(\frac{N_{12} t_{\frac{\alpha_{1}-2}{1}}}{\Gamma\left(\alpha_{1}\right)}\right.\right.\right. \\
& \left.-\frac{N_{22} \lambda_{2} G_{2}\left(\eta_{2}-\alpha_{2}+\theta_{2}\right) \stackrel{\theta_{2}-1}{\underline{B}} \mathcal{B}_{2}}{\Gamma\left(\theta_{2}\right)}\right)+\frac{M_{12} e^{-m_{2} t_{2}}}{\Gamma\left(\alpha_{1}\right)}\left(t_{2}+\rho_{2}\right) \underline{\rho_{2}} \sum_{s=0}^{t_{1}-\alpha_{1}}\left(t_{1}-\sigma(s)\right) \frac{\alpha_{1}-1}{} \times \\
& \left.e^{-2 m_{2} s}-\frac{M_{22} G_{2}\left(\eta_{2}-\alpha_{2}+\theta_{2}-1\right) \underline{\theta_{2}-1} e^{-m_{2}\left(t_{1}+2 t_{2}\right)}\left(t_{2}+\rho_{2}\right) \underline{\rho_{2}}}{\Gamma\left(\theta_{2}\right)}\right]+t \frac{\alpha_{1}-1}{1} \times \\
& {\left[\left(t_{1}+\rho_{1}\right)^{\rho_{1}}\left[\left(t_{2}+\rho_{2}\right)^{\rho_{2}}\right]^{2} e^{-n_{2}\left(t_{2}+1\right)}\left(\frac{N_{22} t_{2}^{\alpha_{2}-2}}{\Gamma\left(\alpha_{2}\right)}-\frac{N_{12} \lambda_{1} G_{1}\left(\eta_{1}-\alpha_{1}+\theta_{1}\right) \frac{\theta_{1}-1}{\Gamma\left(\theta_{1}\right)} \mathcal{B}_{1}}{\Gamma}\right)\right.} \\
& +\frac{M_{22} e^{-m_{2} t_{1}}}{\Gamma\left(\alpha_{2}\right)} \sum_{s=0}^{t_{2}-\alpha_{2}}\left(t_{2}-\sigma(s)\right) \frac{\alpha_{2}-1}{2} e^{-2 m_{2} s}\left(t_{2}+\rho_{2}\right) \frac{\rho_{2}}{} \\
& \left.\left.-\frac{M_{22} G_{1}\left(\eta_{1}-\alpha_{1}+\theta_{1}-1\right) \underline{\theta_{1}-1} e^{-m_{2}\left(t_{2}+2 t_{2}\right)}}{\Gamma\left(\theta_{1}\right)}\right]+\frac{N_{22}}{\Gamma\left(\alpha_{2}\right)}\left[\left(t_{2}+\rho_{2}\right) \frac{\rho_{2}}{2}\right]^{2} e^{-n_{2}\left(t_{2}+1\right)} t_{2}^{\frac{\alpha_{2}-2}{2}}\right\} \\
& +\frac{M_{22} e^{-m_{2} t_{1}}}{\Gamma\left(\alpha_{2}\right)} \sum_{s=0}^{t_{2}-\alpha_{2}}\left(t_{2}-\sigma(s)\right) \underline{\alpha_{2}-1} e^{-2 m_{2} s}\left(t_{2}+\rho_{2}\right)^{\rho_{2}} .
\end{aligned}
$$

It is clear that $\left(\omega_{1}, \omega_{2}\right) \in \mathcal{U}$. For $\ell>0$, we define:

$$
\Xi_{\ell}=\left\{\left(u_{1}, u_{2}\right) \in \mathcal{U}:\left\|\left(u_{1}, u_{2}\right)-\left(\omega_{1}, \omega_{2}\right)\right\|_{\mathcal{U}} \leq \ell\right\} .
$$

For $\left(u_{1}, u_{2}\right) \in \Xi_{\ell}$, we have:

$$
\begin{aligned}
\left\|\left(u_{1}, u_{2}\right)\right\|_{\mathcal{U}} & \leq\left\|\left(u_{1}, u_{2}\right)-\left(\omega_{1}, \omega_{2}\right)\right\|_{\mathcal{U}}+\left\|\left(\omega_{1}, \omega_{2}\right)\right\|_{\mathcal{U}} \leq \ell+\left\|\left(\omega_{1}, \omega_{2}\right)\right\|_{\mathcal{U}}, \\
\left\|\left(u_{1}, u_{2}\right)\right\|_{\mathcal{U}} & =\max \left\{\left\|\left(u_{1}, u_{2}\right)\right\|_{\mathcal{U}_{1}},\left\|\left(u_{1}, u_{2}\right)\right\|_{\mathcal{U}_{2}}\right\} \leq \ell+\left\|\left(\omega_{1}, \omega_{2}\right)\right\|_{\mathcal{U}} .
\end{aligned}
$$

Using the conditions $\left(H_{1}\right)-\left(H_{2}\right)$, together with the procedure employed in Lemma 5, we have:

$$
\begin{aligned}
& \left|F_{i}\left(t_{1}, t_{2}, \Delta^{\beta_{i}} u_{i}, u_{j}\right)-M_{i 1}\left(t_{2}+{ }_{i 1} \rho_{2}\right) \frac{i 1 \rho_{2}}{2} e^{-m_{i 1}\left(t_{1}+t_{2}\right)}\right| \\
\leq & \left\{M_{i 2}\left(t_{2}+{ }_{i 2} \rho_{2}\right) \frac{i 2 \rho_{2}}{2} e^{-m_{i 2}\left(t_{1}+t_{2}\right)}\right.
\end{aligned}
$$




$$
\left.+M_{i 3}\left(t_{2}+{ }_{i 3} \rho_{2}\right) \frac{i 3 \rho_{2}}{\underline{e}} e^{-m_{i 3}\left(t_{1}+t_{2}\right)}\right\}\left\|\left(u_{1}, u_{2}\right)\right\|_{\mathcal{U}}
$$

and:

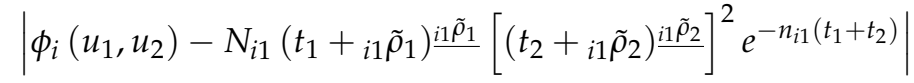

$$
\begin{aligned}
& =\left\{N_{i 2}\left(t_{1}+{ }_{i 2} \tilde{\rho}_{1}\right) \frac{i 2 \tilde{\rho}_{1}}{2}\left[\left(t_{2}+{ }_{i 2} \tilde{\rho}_{2}\right) \frac{i 2 \tilde{\rho}_{2}}{2}\right]^{2} e^{-n_{i 2}\left(t_{1}+t_{2}\right)}\right. \\
& \left.+N_{i 3}\left(t_{1}+{ }_{i 3} \tilde{\rho}_{1}\right) \frac{i 3 \tilde{\rho}_{1}}{\tilde{\rho}_{1}}\left[\left(t_{2}+{ }_{i 3} \tilde{\rho}_{2}\right) \frac{i 3 \tilde{\rho}_{2}}{2}\right]^{-n_{i 3}\left(t_{1}+t_{2}\right)}\right\}\left\|\left(u_{1}, u_{2}\right)\right\|_{\mathcal{U}}
\end{aligned}
$$

Therefore, we obtain:

$$
\chi\left|\left(\mathcal{F}_{i}\left(u_{i}, u_{j}\right)\right)\left(t_{1}, t_{2}\right)-\omega_{i}\left(t_{1}, t_{2}\right)\right| \leq\left(\ell+\left\|\left(\omega_{1}, \omega_{2}\right)\right\| \mathcal{U}\right) \Psi_{i} .
$$

Furthermore, we have:

$$
\begin{aligned}
& \chi\left|\Delta^{\beta_{i}}\left(\mathcal{F}_{i}\left(u_{i}, u_{j}\right)\right)\left(t_{i}-\beta_{i}+1, t_{j}\right)-\Delta^{\beta_{i}} \omega_{i}\left(t_{i}-\beta_{i}+1, t_{j}\right)\right| \\
\leq & \left(\ell+\left\|\left(\omega_{1}, \omega_{2}\right)\right\|_{\mathcal{U}}\right) \Psi_{i} .
\end{aligned}
$$

Hence, it follows that:

$$
\left\|\left(\mathcal{F}_{i}\left(u_{1}, u_{2}\right)\right)-\omega_{i}\right\|_{\mathcal{U}_{i}} \leq\left(\ell+\left\|\left(\omega_{1}, \omega_{2}\right)\right\|_{\mathcal{U}}\right) 2 \Psi_{i}
$$

Therefore,

$$
\left\|\left(\mathcal{F}\left(u_{1}, u_{2}\right)\right)-\left(\omega_{1}, \omega_{2}\right)\right\|_{\mathcal{U}} \leq\left(\ell+\left\|\left(\omega_{1}, \omega_{2}\right)\right\|_{\mathcal{U}}\right) 2 \max \left\{\Psi_{1}, \Psi_{2}\right\} .
$$

Choosing:

$$
\ell \geq \frac{\left\|\left(\omega_{1}, \omega_{2}\right)\right\|_{\mathcal{U}} 2 \max \left\{\Psi_{1}, \Psi_{2}\right\}}{1-2 \max \left\{\Psi_{1}, \Psi_{2}\right\}}
$$

and for $\left(u_{1}, u_{2}\right) \in \Xi_{\ell}$, we consequently obtain:

$$
\left\|\left(\mathcal{F}\left(u_{1}, u_{2}\right)\right)-\left(\omega_{1}, \omega_{2}\right)\right\|_{\mathcal{U}} \leq \ell .
$$

From the Schauder fixed point theorem, this implies that $\mathcal{F}$ has a fixed point $\left(u_{1}, u_{2}\right) \in \Xi_{\ell}$, which is a bounded solution of the problems (5) and (6). The proof is complete.

\section{Example}

In order to illustrate our result, we consider the following fractional sum boundary value problem:

$$
\begin{aligned}
\Delta^{\frac{3}{2}} u_{1}(t)= & \frac{2}{3}\left(t+\frac{4}{3}\right) e^{-(12 t+5)}+\frac{\left(t+\frac{5}{3}\right)^{\frac{4}{3}} e^{-\left(25 t+\frac{31}{3}\right)} u_{2}\left(t+\frac{1}{3}\right)}{4000\left(t+\frac{301}{3}\right)^{2}\left(1+\cos ^{2} u_{2} \pi\right)} \\
& +\frac{\left(t+\frac{11}{6}\right)^{\frac{3}{2}} e^{-\left[(12 t+5)+\left(t+\frac{1}{2}\right) \pi\right]} \Delta^{\frac{1}{3}} u_{1}\left(t+\frac{4}{3}\right)}{5000 e+10 \cos ^{2}\left(t+\frac{1}{2}\right) \pi}, t \in \mathbb{N}_{0}
\end{aligned}
$$




$$
\begin{aligned}
\Delta^{\frac{4}{3}} u_{2}(t)= & \frac{1}{2}\left(t+\frac{4}{3}\right) e^{-(12 t+5)}+\frac{\left(t+\frac{5}{3}\right)^{\frac{4}{3}} e^{-\left(25 t+\frac{21}{3}\right)} u_{1}\left(t+\frac{1}{2}\right)}{1000\left(e^{\left(t+\frac{1}{2}\right)}+10\right)^{2}} \\
& +\frac{\left(t+\frac{11}{6}\right)^{\frac{3}{2}} e^{-(12 t+5)} \arctan \left(\cos ^{2}\left(t+\frac{1}{3}\right) \pi\right) \Delta^{\frac{3}{4}} u_{2}\left(t_{2}+\frac{7}{12}\right)}{1000 \pi\left(t+\frac{10}{3}\right)^{2}}, t \in \mathbb{N}_{0} \\
u_{1}\left(-\frac{1}{2}\right)= & \phi_{1}\left(u_{1}, u_{2}\right)=\frac{\left|u_{1}\right|}{2000 e^{3}} \cos ^{2}\left|\pi u_{1}\right|+\frac{\left|u_{2}\right|\left|u_{2}^{2}+2\right| \frac{1-\left|u_{2}^{2}+2\right|}{4000 \pi^{2}\left(u_{2}^{2}+e\right)}}{2000 \pi\left(u_{1}^{2}+\pi\right)}, \\
u_{2}\left(-\frac{2}{3}\right)= & \phi_{2}\left(u_{1}, u_{2}\right)=\frac{\left|u_{2}\right|}{5000 e^{2}} \sin ^{2}\left|\pi u_{2}\right|+\frac{\left|u_{1}\right|\left|u_{1}^{2}+3\right| \frac{1-\left|u_{1}^{2}+3\right|}{2}}{\lim _{t \longrightarrow \infty} u_{1}\left(t-\frac{1}{2}\right)=} \frac{1}{2} \Delta^{-\frac{1}{4}}(12 e+\cos (4))^{2} u_{2}(4) \\
\lim _{t \longrightarrow \infty} u_{2}\left(t-\frac{2}{3}\right)= & \frac{3}{4} \Delta^{-\frac{2}{3}}\left(10 e-\sin \left(\frac{15}{4}\right)\right)^{3} u_{1}\left(\frac{15}{4}\right) .
\end{aligned}
$$

Here, $\alpha_{1}=\frac{3}{2}, \alpha_{2}=\frac{4}{3}, \beta_{1}=\frac{1}{3}, \beta_{2}=\frac{3}{4}, \gamma_{1}=\frac{3}{4}, \gamma_{2}=\frac{5}{6}, \theta_{1}=\frac{1}{4}, \theta_{2}=\frac{2}{3}, \eta_{1}=\frac{7}{2}, \eta_{2}=\frac{10}{3}, \lambda_{1}=$ $\frac{1}{2}, \lambda_{2}=\frac{3}{4}, T=4, g_{1}\left(t_{1}\right)=\left(10 e-\sin t_{1}\right)^{3}, g_{2}\left(t_{2}\right)=\left(12 e+\cos t_{2}\right)^{2}$, and:

$$
\begin{aligned}
F_{1}\left(t_{1}, t_{2}, \Delta^{\frac{1}{3}} u_{1}\left(t_{1}+\frac{2}{3}\right), u_{2}\left(t_{2}\right)\right) & =\frac{2}{3}\left(t_{2}+1\right) e^{-6\left(t_{1}+t_{2}\right)}+\frac{\left(t_{2}+\frac{4}{3}\right)^{\frac{4}{3}} e^{-\left[12\left(t_{1}+t_{2}\right)+t_{2}\right]}}{4000\left(t_{2}+10\right)^{2}\left(1+\cos ^{2} u_{2} \pi\right)} \\
& +\frac{\left(t_{2}+\frac{3}{2}\right)^{\frac{3}{2}} e^{-\left[6\left(t_{1}+t_{2}\right)+t_{1} \pi\right]} \Delta^{\frac{1}{3}} u_{1}\left(t_{1}+\frac{2}{3}\right)}{5000 e+10 \cos ^{2} t_{1} \pi} \\
F_{2}\left(t_{1}, t_{2}, \Delta^{\frac{3}{4}} u_{2}\left(t_{2}+\frac{1}{4}\right), u_{1}\left(t_{1}\right)\right) & \left.=\frac{1}{2}\left(t_{2}+1\right) e^{-6\left(t_{1}+t_{2}\right)}+\frac{\left(t_{2}+\frac{4}{3}\right)^{\frac{4}{3}} e^{-\left[12\left(t_{1}+t_{2}\right)+t_{1}\right]}}{1000\left(e_{1} t_{1}+10\right)^{2}}\right) \\
& +\frac{\left(t_{2}+\frac{3}{2}\right)^{\frac{3}{2}} e^{-6\left(t_{1}+t_{2}\right)} \arctan \left(\cos ^{2} t_{2} \pi\right) \Delta^{\frac{3}{4}} u_{2}\left(t_{2}+\frac{1}{4}\right)}{1000 \pi\left(t_{2}+3\right)^{2}} .
\end{aligned}
$$

Choose $\rho_{1}=1, \rho_{2}=2,{ }_{i 1} \rho_{1}={ }_{i 1} \tilde{\rho}_{1}=\frac{1}{2},{ }_{i 2} \rho_{1}={ }_{i 2} \tilde{\rho}_{1}=\frac{2}{3},{ }_{i 3} \rho_{1}={ }_{i 3} \tilde{\rho}_{1}=\frac{3}{4}$ ${ }_{i 1} \rho_{2}=1,{ }_{i 2} \rho_{2}=\frac{3}{2}, i_{33} \rho_{2}=\frac{4}{3}, m_{i 1}=n_{i 1}=6, m_{i 2}=n_{i 2}=6, m_{i 3}=n_{i 3}=12$, where $\rho_{i}>\max \left\{\beta_{1}-\alpha_{1}, \beta_{2}-\alpha_{2}\right\},{ }_{i p} \rho_{1} \in(-1,1),{ }_{i p} \rho_{2} \in(-1,2)$ for $i=1,2$ and $p=1,2,3$.

Let $t_{1} \in \mathbb{N}_{-\frac{1}{2}, \frac{11}{2}}, t_{2} \in \mathbb{N}_{-\frac{2}{3}, \frac{16}{3}}$ and $\chi=\frac{\left(t_{1}-\frac{1}{2}\right)^{\frac{1 / 2}{2}}\left(t_{2}-\frac{1}{3}\right)^{2 / 3}}{1+t_{1}^{3} t_{\frac{4}{2}}^{4}}$. Since:

$$
\begin{aligned}
& \left|F_{1}\left(t_{1}, t_{2}, \frac{1}{\chi} \Delta^{\frac{1}{3}} u_{1}, \frac{1}{\chi} u_{2}\right)-\frac{2}{3}\left(t_{2}+1\right) e^{-6\left(t_{1}+t_{2}\right)}\right| \\
\leq & \frac{1}{361000}\left(t_{2}+\frac{4}{3}\right)^{\frac{4}{3}} e^{-12\left(t_{1}+t_{2}\right)}\left|u_{2}\right|+\frac{1}{5000 e^{10}+10}\left(t_{2}+\frac{3}{2}\right)^{\frac{3}{2}} e^{-6\left(t_{1}+t_{2}\right)}\left|\Delta^{\frac{1}{3}} u_{1}\right|, \\
& \left|F_{2}\left(t_{1}, t_{2}, \frac{1}{\chi} \Delta^{\frac{3}{4}} u_{2}, \frac{1}{\chi} u_{1}\right)-\frac{1}{2}\left(t_{2}+1\right) e^{-6\left(t_{1}+t_{2}\right)}\right| \\
\leq & \frac{1}{121000}\left(t_{2}+\frac{4}{3}\right)^{\frac{4}{3}} e^{-12\left(t_{1}+t_{2}\right)}\left|u_{2}\right|+\frac{9}{196000}\left(t_{2}+\frac{3}{2}\right)^{\frac{3}{2}} e^{-6\left(t_{1}+t_{2}\right)}\left|\Delta^{\frac{3}{4}} u_{2}\right|,
\end{aligned}
$$

we find that $\left(H_{1}\right)$ holds with $M_{11}=0.666, M_{12}=9.080, M_{13}=2.770$ and $M_{21}=0.500, M_{22}=$ $0.000046, M_{23}=0.0000083$.

Furthermore, we obtain:

$$
\left|\phi_{1}\left(\frac{1}{\chi} u_{1}, \frac{1}{\chi} u_{2}\right)-\frac{2}{5}\left(t_{1}+\frac{1}{2}\right)^{\frac{1}{2}}\left(t_{2}+\frac{1}{2}\right) e^{-6\left(t_{1}+t_{2}\right)}\right|
$$




$$
\begin{aligned}
\leq & \frac{1}{2000 e^{3}}\left(t_{1}+\frac{2}{3}\right)^{\frac{2}{3}}\left(t_{2}+\frac{2}{3}\right)^{\frac{4}{3}} e^{-6\left(t_{1}+t_{2}\right)}\left\|u_{1}\right\|+\frac{1}{4000 \pi^{2}}\left(t_{1}+\frac{3}{4}\right)^{\frac{3}{4}}\left(t_{2}+\frac{3}{4}\right)^{\frac{3}{2}} \times \\
& e^{-3\left(t_{1}+t_{2}\right)}\left\|u_{2}\right\|, \\
& \left|\phi_{2}\left(\frac{1}{\chi} u_{1}, \frac{1}{\chi} u_{2}\right)-\frac{5}{6}\left(t_{1}+\frac{1}{2}\right)^{\frac{1}{2}}\left(t_{2}+\frac{1}{2}\right) e^{-6\left(t_{1}+t_{2}\right)}\right| \\
\leq & \frac{1}{2000 \pi}\left(t_{1}+\frac{2}{3}\right)^{\frac{2}{3}}\left(t_{2}+\frac{2}{3}\right)^{\frac{4}{3}} e^{-6\left(t_{1}+t_{2}\right)}\left\|u_{1}\right\|+\frac{1}{500 e^{2}}\left(t_{1}+\frac{3}{4}\right)^{\frac{3}{4}}\left(t_{2}+\frac{3}{4}\right)^{\frac{3}{2}} \times \\
& e^{-3\left(t_{1}+t_{2}\right)}\left\|u_{2}\right\|,
\end{aligned}
$$

and $(10 e-1)^{3}<g_{1}\left(t_{1}\right)<(10 e+1)^{3}$ and $(12 e-1)^{2}<g_{2}\left(t_{2}\right)<(12 e+1)^{2}$.

Thus, $\left(H_{2}\right),(H 3)$ hold with $N_{11}=0.4, N_{12}=0.0000249, N_{13}=0.0000253, N_{21}=0.833$, $N_{22}=0.000159, N_{23}=0.000271, g_{1}=17949.37, g_{2}=999.79, G_{1}=22384.80$, and $G_{2}=1130.26$.

Finally, we find that:

$$
\begin{gathered}
\tilde{\Omega}_{11}=2313.238, \tilde{\Omega}_{12}=319.647, \tilde{\Omega}_{13}=27053.522, \tilde{\Omega}_{14}=2597.063, \\
\tilde{\Omega}_{21}=0.0212, \tilde{\Omega}_{22}=1.1403, \tilde{\Omega}_{23}=0.0198, \tilde{\Omega}_{24}=1.5093 .
\end{gathered}
$$

Therefore, we have:

$$
\Psi_{1}+\Psi_{2}=0.00057+0.4692=0.4698<1 .
$$

Hence, by Theorem 4, this boundary value problem has at least one solution.

Author Contributions: These authors contributed equally to this work.

Funding: This research was funded by King Mongkut's University of Technology, North Bangkok, Contract No. KMUTNB-ART-60-38.

Acknowledgments: The last author of this research was supported by Suan Dusit University.

Conflicts of Interest: The authors declare no conflicts of interest regarding the publication of this paper.

\section{References}

1. Wu, G.C.; Baleanu, D. Discrete fractional logistic map and its chaos. Nonlinear Dyn. 2014, 75, $283-287$. [CrossRef]

2. Wu, G.C.; Baleanu, D. Chaos synchronization of the discrete fractional logistic map. Signal Process. 2014, 102, 96-99. [CrossRef]

3. Goodrich, C.S.; Peterson, A.C. Discrete Fractional Calculus; Springer: New York, NY, USA, 2015.

4. Atici, F.M.; Eloe, P.W. A transform method in discrete fractional calculus. Int. J. Differ. Equ. 2007, 2, $165-176$.

5. Atici, F.M.; Eloe, P.W. Initial value problems in discrete fractional calculus. Proc. Am. Math. Soc. 2009, 137, 981-989. [CrossRef]

6. Atici, F.M.; Eloe, P.W. Two-point boundary value problems for finite fractional difference equations. J. Differ. Equ. Appl. 2011, 17, 445-456. [CrossRef]

7. Abdeljawad, T. On Riemann and Caputo fractional differences. Comput. Math. Appl. 2011, 62, 1602-1611. [CrossRef]

8. Abdeljawad, T. Dual identities in fractional difference calculus within Riemann. Adv. Differ. Equ. 2013, 2013, 36. [CrossRef]

9. Abdeljawad, T. On delta and nabla Caputo fractional differences and dual identities. Discret. Dyn. Nat. Soc. 2013, 2013, 406910. [CrossRef]

10. Abdeljawad, T.; Baleanu, D. Fractional differences and integration by parts. J. Comput. Anal. Appl. 2011, 13, 574-582.

11. Holm, M. Sum and difference compositions in discrete fractional calculus. Cubo 2011, 13, 153-184. [CrossRef]

12. Anastassiou, G. Foundations of nabla fractional calculus on time scales and inequalities. Comput. Math. Appl. 2010, 59, 3750-3762. [CrossRef] 
13. Jia, B.; Erbe, L.; Peterson, A. Two monotonicity results for nabla and delta fractional differences. Arch. Math. 2015, 104, 589-597. [CrossRef]

14. Jia, B.; Erbe, L.; Peterson, A. Convexity for nabla and delta fractional differences. J. Differ. Equ. Appl. 2015, 21, 360-373.

15. Cermák, J.; Kisela, T.; Nechvátal, L. Stability and asymptotic properties of a linear fractional difference equation. Adv. Differ. Equ. 2012, 2012, 122. [CrossRef]

16. Jarad, F.; Abdeljawad, T.; Baleanu, D.; Biçen, K. On the stability of some discrete fractional nonautonomous systems. Abstr. Appl. Anal. 2012, 2012, 476581. [CrossRef]

17. Mozyrska, D.; Wyrwas, M. The Z-transform method and delta type fractional difference operators. Discret. Dyn. Nat. Soc. 2015, 2015, 12. [CrossRef]

18. Mozyrska, D.; Wyrwas, M. Explicit criteria for stability of fractional $h$-difference two-dimensional systems. Int. J. Dyn. Control 2017, 5, 4-9. [CrossRef]

19. Ferreira, R.A.C.; Torres, D.F.M. Fractional $h$-difference equations arising from the calculus of variations. Appl. Anal. Discret. Math. 2011, 5, 110-121. [CrossRef]

20. Ferreira, R.A.C. Existence and uniqueness of solution to some discrete fractional boundary value problems of order less than one. J. Differ. Equ. Appl. 2013, 19, 712-718. [CrossRef]

21. Ferreira, R.A.C.; Goodrich, C.S. Positive solution for a discrete fractional periodic boundary value problem. Dyn. Contin. Discret. Impuls. Syst. Ser. A Math. Anal. 2012, 19, 545-557.

22. Goodrich, C.S. Existence and uniqueness of solutions to a fractional difference equation with nonlocal conditions. Comput. Math. Appl. 2011, 61, 191-202. [CrossRef]

23. Goodrich, C.S. On a discrete fractional three-point boundary value problem. J. Differ. Equ. Appl. 2012, 18, 397-415. [CrossRef]

24. Goodrich, C.S. A convexity result for fractional differences. Appl. Math. Lett. 2014, 35, 58-62. [CrossRef]

25. Goodrich, C.S. The relationship between sequential fractional difference and convexity. Appl. Anal. Discret. Math. 2016, 10, 345-365. [CrossRef]

26. Dahal, R.; Goodrich, C.S. A monotonicity result for discrete fractional difference operators. Arch. Math. 2014, 102, 293-299. [CrossRef]

27. Erbe, L.; Goodrich, C.S.; Jia, B.; Peterson, A. Survey of the qualitative properties of fractional difference operators: Monotonicity, convexity, and asymptotic behavior of solutions. Adv. Differ. Equ. 2016, 2016, 43. [CrossRef]

28. Chen, F.; Luo, X.; Zhou, Y. Existence results for nonlinear fractional difference equation. Adv. Differ. Equ. 2011, 2011, 713201. [CrossRef]

29. Chen, F.; Zhou, Y. Existence and Ulam stability of solutions for discrete fractional boundary value problem. Discret. Dyn. Nat. Soc. 2013, 2013, 459161. [CrossRef]

30. Chen, Y.; Tang, X. Thee difference between a class of discrete fractional and integer order boundary value problems. Commun. Nonlinear Sci. 2014, 19, 4057-4067. [CrossRef]

31. Lv, W. Solvability for discrete fractional boundary value problems with a p-laplacian operator. Discret. Dyn. Nat. Soc. 2013, 2013, 679290. [CrossRef]

32. Lv, W. Solvability for a discrete fractional three-point boundary value problem at resonance. Abstr. Appl. Anal. 2014, 2014, 601092. [CrossRef]

33. Lv, W.; Feng, J. Nonlinear discrete fractional mixed type sum-difference equation boundary value problems in Banach spaces. Adv. Differ. Equ. 2014, 2014, 184. [CrossRef]

34. Chen, H.Q.; Cui, Y.Q.; Zhao, X.L. Multiple solutions to fractional difference boundary value problems. Abstr. Appl. Anal. 2014, 2014, 879380. [CrossRef]

35. Chen, H.Q.; Jin, Z.; Kang, S.G. Existence of positive solutions for Caputo fractional difference equation. Adv. Differ. Equ. 2015, 2015, 44. [CrossRef]

36. Kang, S.G.; Li, Y.; Chen, H.Q. Positive solutions to boundary value problems of fractional difference equations with nonlocal conditions. Adv. Differ. Equ. 2014, 2014, 7. [CrossRef]

37. Dong, W.; Xu, J.; Regan, D.O. Solutions for a fractional difference boundary value problem. Adv. Differ. Equ. 2013, 2013, 319. [CrossRef]

38. Sitthiwirattham, T.; Tariboon, J.; Ntouyas, S.K. Existence Results for fractional difference equations with three-point fractional sum boundary conditions. Discret. Dyn. Nat. Soc. 2013, 2013, 104276. [CrossRef]

39. Sitthiwirattham, T.; Tariboon, J.; Ntouyas, S.K. Boundary value problems for fractional difference equations with three-point fractional sum boundary conditions. Adv. Differ. Equ. 2013, 2013, 296. [CrossRef] 
40. Sitthiwirattham, T. Existence and uniqueness of solutions of sequential nonlinear fractional difference equations with three-point fractional sum boundary conditions. Math. Methods Appl. Sci. 2015, 38, 2809-2815. [CrossRef]

41. Sitthiwirattham, T. Boundary value problem for $p$-Laplacian Caputo fractional difference equations with fractional sum boundary conditions. Math. Methods Appl. Sci. 2016, 39, 1522-1534. [CrossRef]

42. Chasreechai, S.; Kiataramkul, C.; Sitthiwirattham, T. On nonlinear fractional sum-difference equations via fractional sum boundary conditions involving different orders. Math. Probl. Eng. 2015, 2015, 519072. [CrossRef]

43. Reunsumrit, J.; Sitthiwirattham, T. Positive solutions of three-point fractional sum boundary value problem for Caputo fractional difference equations via an argument with a shift. Positivity 2016, 20, 861-876. [CrossRef]

44. Reunsumrit, J.; Sitthiwirattham, T. On positive solutions to fractional sum boundary value problems for nonlinear fractional difference equations. Math. Methods Appl. Sci. 2016, 39, 2737-2751. [CrossRef]

45. Soontharanon, J.; Jasthitikulchai, N.; Sitthiwirattham, T. Nonlocal Fractional Sum Boundary Value Problems for Mixed Types of Riemann-Liouville and Caputo Fractional Difference Equations. Dyn. Syst. Appl. 2016, 25, 409-414.

46. Laoprasittichok, S.; Sitthiwirattham, T. On a Fractional Difference-Sum Boundary Value Problems for Fractional Difference Equations Involving Sequential Fractional Differences via Different Orders. J. Comput. Anal. Appl. 2017, 23, 1097-1111.

47. Kaewwisetkul, B.; Sitthiwirattham, T. On Nonlocal Fractional Sum-Difference Boundary Value Problems for Caputo Fractional Functional Difference Equations with Delay. Adv. Differ. Equ. 2017, 2017, 219. [CrossRef]

48. Ahmad, B.; Ntouyas, S.K.; Alsaedi, A. On a coupled system of fractional differential equations with coupled nonlocal and integral boundary conditions. Chaos Soliton Fractals 2016, 83, 234-241. [CrossRef]

49. Ahmad, B.; Ntouyas, S.K. Existence results for a coupled system of Caputo type sequential fractional differential equations with nonlocal integral boundary conditions. Appl. Math. Comput. 2015, 266, 615-622. [CrossRef]

50. Henderson, J.; Luca, R. Nonexistence of positive solutions for a system of coupled fractional boundary value problems. Bound. Value Probl. 2015, 2015, 138. [CrossRef]

51. Henderson, J.; Luca, R.; Tudorache, A. On a system of fractional differential equations with coupled integral boundary conditions. Fract. Calc. Appl. Anal. 2015, 18, 361-386. [CrossRef]

52. Wang, J.R.; Zhang, Y. Analysis of fractional order differential coupled systems. Math. Methods Appl. Sci. 2015, 38, 3322-3338. [CrossRef]

53. $\mathrm{Su}, \mathrm{X}$. Boundary value problem for a coupled system of nonlinear fractional differential equations. Appl. Math. Lett. 2009, 22, 64-69. [CrossRef]

54. Ascione, G.; Leonenko, N.; Pirozzi, E. Fractional Queues with Catastrophes and Their Transient Behaviour. Mathematics 2018, 6, 159. [CrossRef]

55. Pan, Y.; Han, Z.; Sun, S.; Zhao, Y. The Existence of Solutions to a System of Discrete Fractional Boundary Value Problems. Abstr. Appl. Anal. 2012, 2012, 707631. [CrossRef]

56. Goodrich, C.S. Existence of a positive solution to a system of discrete fractional boundary value problems. Appl. Math. Comput. 2011, 217, 4740-4753. [CrossRef]

57. Dahal, R.; Duncan, D.; Goodrich, C.S. Systems of semipositone discrete fractional boundary value problems. J. Differ. Equ. Appl. 2014, 20, 473-491. [CrossRef]

58. Goodrich, C.S. Systems of discrete fractional boundary value problems with nonlinearities satisfying no growth conditions. J. Differ. Equ. Appl. 2015, 21, 437-453. [CrossRef]

59. Goodrich, C.S. Coupled systems of boundary value problems with nonlocal boundary conditions. Appl. Math. Lett. 2015, 41,17-22. [CrossRef]

60. Kunnawuttipreechachan, E.; Promsakon, C.; Sitthiwirattham, T. Nonlocal fractional sum boundary value problems for a coupled system of fractional sum-difference equations. Dyn. Syst. Appl. 2019, 28, 73-92. [CrossRef]

61. Griffel, D.H. Applied Functional Analysis; Ellis Horwood Publishers: Chichester, UK, 1981.

(C) 2019 by the authors. Licensee MDPI, Basel, Switzerland. This article is an open access article distributed under the terms and conditions of the Creative Commons Attribution (CC BY) license (http://creativecommons.org/licenses/by/4.0/). 